\title{
POLYNOMIAL INEQUALITIES ON THE HAMMING CUBE
}

\author{
ALEXANDROS ESKENAZIS AND PAATA IVANISVILI
}

\begin{abstract}
Let $\left(X,\|\cdot\|_{X}\right)$ be a Banach space. The purpose of this article is to systematically investigate dimension independent properties of vector valued functions $f:\{-1,1\}^{n} \rightarrow X$ on the Hamming cube whose spectrum is bounded above or below. Our proofs exploit contractivity properties of the heat flow, induced by the geometry of the target space $\left(X,\|\cdot\|_{X}\right)$, combined with duality arguments and suitable tools from approximation theory and complex analysis. We obtain a series of improvements of various well-studied estimates for functions with bounded spectrum, including moment comparison results for low degree Walsh polynomials and Bernstein-Markov type inequalities, which constitute discrete vector valued analogues of Freud's inequality in Gauss space (1971). Many of these inequalities are new even for scalar valued functions. Furthermore, we provide a short proof of Mendel and Naor's heat smoothing theorem (2014) for functions in tail spaces with values in spaces of nontrivial type and we also prove a dual lower bound on the decay of the heat semigroup acting on functions with spectrum bounded from above. Finally, we improve the reverse Bernstein-Markov inequalities of Meyer (1984) and Mendel and Naor (2014) for functions with narrow enough spectrum and improve the bounds of Filmus, Hatami, Keller and Lifshitz (2016) on the $\ell_{p}$ sums of influences of bounded functions for $p \in\left(1, \frac{4}{3}\right)$.
\end{abstract}

2010 Mathematics Subject Classification. Primary: 42C10; Secondary: 41A17, 41A63, $46 \mathrm{~B} 07$.

Key words. Hamming cube, heat semigroup, hypercontractivity, Bernstein-Markov inequality, moment comparison.

\section{INTRODUCTION}

Fix $n \in \mathbb{N}$ and let $\left(X,\|\cdot\|_{X}\right)$ be a Banach space. If $p \in[1, \infty)$, the vector valued $L_{p}$ norm of a function $f:\{-1,1\}^{n} \rightarrow X$ is defined as

$$
\|f\|_{L_{p}\left(\{-1,1\}^{n} ; X\right)} \stackrel{\text { def }}{=}\left(\frac{1}{2^{n}} \sum_{\varepsilon \in\{-1,1\}^{n}}\|f(\varepsilon)\|_{X}^{p}\right)^{1 / p} .
$$

As usual, we denote $\|f\|_{L_{\infty}\left(\{-1,1\}^{n} ; X\right)} \stackrel{\text { def }}{=} \max _{\varepsilon \in\{-1,1\}^{n}}\|f(\varepsilon)\|_{X}$. For a subset $A \subseteq\{1, \ldots, n\}$ the Walsh function $w_{A}:\{-1,1\}^{n} \rightarrow\{-1,1\}$ is the Boolean function given by $w_{A}(\varepsilon)=\prod_{i \in A} \varepsilon_{i}$, where $\varepsilon=\left(\varepsilon_{1}, \ldots, \varepsilon_{n}\right) \in\{-1,1\}^{n}$. Every function $f:\{-1,1\}^{n} \rightarrow X$ admits an expansion of the form

$$
f=\sum_{A \subseteq\{1, \ldots, n\}} \widehat{f}(A) w_{A}
$$

where

$$
\widehat{f}(A) \stackrel{\text { def }}{=} \frac{1}{2^{n}} \sum_{\delta \in\{-1,1\}^{n}} f(\delta) w_{A}(\delta) \in X .
$$

For $i \in\{1, \ldots, n\}$ the $i$-th partial derivative of such a function $f$ is given by

$$
\partial_{i} f(\varepsilon) \stackrel{\text { def }}{=} \frac{f(\varepsilon)-f\left(\varepsilon_{1}, \ldots, \varepsilon_{i-1},-\varepsilon_{i}, \varepsilon_{i+1}, \ldots, \varepsilon_{n}\right)}{2}=\sum_{\substack{A \subseteq\{1, \ldots, n\} \\ i \in A}} \widehat{f}(A) w_{A}(\varepsilon)
$$

P. I. was partially supported by NSF DMS-1856486 and NSF CAREER-1945102. This work was carried out under the auspices of the Simons Algorithms and Geometry (A\&G) Think Tank. 
and satisfies $\partial_{i}^{2} f=\partial_{i} f$. Therefore, the hypercube Laplacian of $f$ is defined as

$$
\Delta f \stackrel{\text { def }}{=} \sum_{i=1}^{n} \partial_{i} f=\sum_{A \subseteq\{1, \ldots, n\}}|A| \widehat{f}(A) w_{A}
$$

Finally, the action of the discrete heat semigroup $\left\{e^{-t \Delta}\right\}_{t \geqslant 0}$ on the function $f$ is given by

$$
\forall t \geqslant 0, \quad e^{-t \Delta} f \stackrel{\text { def }}{=} \sum_{A \subseteq\{1, \ldots, n\}} e^{-t|A|} \widehat{f}(A) w_{A} .
$$

A straightforward calculation shows that the heat semigroup can equivalently be expressed as

$$
e^{-t \Delta} f(\varepsilon)=\frac{1}{2^{n}} \sum_{\delta \in\{-1,1\}^{n}} \sum_{B \subseteq\{1 \ldots, n\}} e^{-t|B|}\left(1-e^{-t}\right)^{n-|B|} f\left(\sum_{j \in B} \varepsilon_{j} e_{j}+\sum_{j \in\{1, \ldots, n\} \backslash B} \delta_{j} e_{j}\right),
$$

where $\left\{e_{1}, \ldots, e_{n}\right\}$ is the orthonormal basis of $\mathbb{R}^{n}$, which by convexity implies that for every $t \geqslant 0$ and $p \in[1, \infty],\left\|e^{-t \Delta} f\right\|_{L_{p}\left(\{-1,1\}^{n} ; X\right)} \leqslant\|f\|_{L_{p}\left(\{-1,1\}^{n} ; X\right)}$. Identity (7) also has a useful probabilistic interpretation. For a fixed $\varepsilon \in\{-1,1\}^{n}$ and $t \geqslant 0$, consider a random vector $\eta \in\{-1,1\}^{n}$ such that each coordinate $\eta_{i}$ is chosen independently to coincide with $\varepsilon_{i}$ with probability $\frac{1+e^{-t}}{2}$ and with $-\varepsilon_{i}$ with probability $\frac{1-e^{-t}}{2}$. Then, the value $e^{-t \Delta} f(\varepsilon)$ is the expectation of the random vector $f(\eta)$.

The main purpose of this paper is to investigate finer contractivity properties of the heat semigroup under suitable assumptions on the spectrum of the function $f$ and the geometry of the target space $\left(X,\|\cdot\|_{X}\right)$. The common feature in the proofs of most of the following results is a duality argument inspired by classical work of Figiel (see [MS86, Theorem 14.6]), which allows the selfimprovement of contractivity properties of the heat semigroup relying on suitable inequalities from classical approximation theory and complex analysis. All Banach spaces in the ensuing discussion will be assumed to be over the field of complex numbers.

In the rest of the introduction, we proceed to describe our results in decreasing order of generality. In Section 1.1, we present estimates for functions with spectrum bounded from above and values in a general Banach space. In Section 1.2, we improve the bounds of Section 1.1 under the additional assumption that the target space $\left(X,\|\cdot\|_{X}\right)$ is $K$-convex (we postpone the relevant definitions until Section 1.2). We also present a new proof of a theorem of Mendel and Naor [MN14] related to the heat smoothing conjecture. Finally, in Section 1.3 we present explicit estimates which hold true for scalar valued functions. These include various Bernstein-Markov type inequalities and their reverses, estimates on the influences of bounded functions and moment comparison results.

1.1. Estimates for a general Banach space. Fix $n \in \mathbb{N}$ and let $\left(X,\|\cdot\|_{X}\right)$ be a Banach space. If $d \in\{0,1 \ldots, n\}$, we say that a function $f:\{-1,1\}^{n} \rightarrow X$ has degree at most $d$ if $\widehat{f}(A)=0$ when $|A|>d$ and we say that $f$ belongs in the $d$-th tail space if $\widehat{f}(A)=0$ when $|A|<d$. Finally, we say that $f$ is $d$-homogeneous if $\widehat{f}(A)=0$ when $|A| \neq d$.

1.1.1. A lower bound on the decay of the heat semigroup. The following theorem establishes a lower bound on the decay of the heat semigroup acting on functions of low degree.

Theorem 1. Fix $n, d \in \mathbb{N}$ with $d \in\{1, \ldots, n\}$ and let $\left(X,\|\cdot\|_{X}\right)$ be a Banach space. For every $p \in[1, \infty]$ and every function $f:\{-1,1\}^{n} \rightarrow X$ of degree at most $d$, we have

$$
\forall t \geqslant 0, \quad\left\|e^{-t \Delta} f\right\|_{L_{p}\left(\{-1,1\}^{n} ; X\right)} \geqslant \frac{1}{T_{d}\left(e^{t}\right)}\|f\|_{L_{p}\left(\{-1,1\}^{n} ; X\right)},
$$

where $T_{d}$ is the d-th Chebyshev polynomial of the first kind. 
We note that a weaker bound in the spirit of Theorem 1, attributed partially to Oleszkiewicz, was established in [FHKL16, Lemma 5.4] (see Remark 18 below for a comparison with Theorem 1).

Using Theorem 1 and the hypercontractivity of the discrete heat semigroup (see [Bon70]), we deduce the following moment comparison for functions of low degree.

Corollary 2. Fix $n, d \in \mathbb{N}$ with $d \in\{1, \ldots, n\}$ and let $\left(X,\|\cdot\|_{X}\right)$ be a Banach space. For every $p>q>1$ and every function $f:\{-1,1\}^{n} \rightarrow X$ of degree at most $d$, we have

$$
\|f\|_{L_{p}\left(\{-1,1\}^{n} ; X\right)} \leqslant T_{d}\left(\sqrt{\frac{p-1}{q-1}}\right)\|f\|_{L_{q}\left(\{-1,1\}^{n} ; X\right)} .
$$

To the extent of our knowledge, the best previously known moment comparison for general vector valued functions of low degree on the discrete cube can be extracted from an argument in the monograph [KW92] of Kwapień and Woyczyński. In Remark 19 below, we quantify their argument and show that the bounds of [KW92, Proposition 6.5.1] are weaker than those of Corollary 2.

Another application of Theorem 1 is a refinement of a celebrated inequality on the discrete cube due to Pisier [Pis86]. In connection with his work on nonlinear type, Pisier showed that for every Banach space $\left(X,\|\cdot\|_{X}\right)$, every $p \in[1, \infty]$ and every function $f:\{-1,1\}^{n} \rightarrow X$, we have

$$
\left\|f-\frac{1}{2^{n}} \sum_{\delta \in\{-1,1\}^{n}} f(\delta)\right\|_{L_{p}\left(\{-1,1\}^{n} ; X\right)} \leqslant(\log n+1)\left(\frac{1}{2^{n}} \sum_{\delta \in\{-1,1\}^{n}}\left\|\sum_{i=1}^{n} \delta_{i} \partial_{i} f\right\|_{L_{p}\left(\{-1,1\}^{n} ; X\right)}^{p}\right)^{1 / p} .
$$

For $X=\mathbb{R}$, the right hand side of (10) is equivalent to the $L_{p}$ norm of the gradient of $f$ due to Khintchine's inequality [Khi23], thus (10) can be understood as a vector valued Poincaré inequality. The dependence on the dimension $n$ in Pisier's inequality (10) for various classes of spaces $X$ is fundamental in investigations in the nonlinear geometry of Banach spaces and the Ribe program (see [Nao12] for a detailed discussion around this topic). For general Banach spaces, Talagrand [Tal93] has proven that the factor $\log n$ in (10) is asymptotically optimal for every $p \in[1, \infty)$, whereas Wagner [Wag00] has shown that when $p=\infty$, Pisier's inequality holds with an absolute constant. Here we show the following refinement of Pisier's inequality for functions of low degree.

Theorem 3. Fix $n, d \in \mathbb{N}$ with $d \in\{1, \ldots, n\}$ and let $\left(X,\|\cdot\|_{X}\right)$ be a Banach space. For every $p \in[1, \infty)$ and every function $f:\{-1,1\}^{n} \rightarrow X$ of degree at most $d$, we have

$$
\left\|f-\frac{1}{2^{n}} \sum_{\delta \in\{-1,1\}^{n}} f(\delta)\right\|_{L_{p}\left(\{-1,1\}^{n} ; X\right)} \leqslant 3(\log d+1)\left(\frac{1}{2^{n}} \sum_{\delta \in\{-1,1\}^{n}}\left\|\sum_{i=1}^{n} \delta_{i} \partial_{i} f\right\|_{L_{p}\left(\{-1,1\}^{n} ; X\right)}^{p}\right)^{1 / p}
$$

and the $\log d$ factor is asymptotically sharp for every $p \in[1, \infty)$.

1.1.2. A Bernstein-Markov type inequality for $\Delta$. A variant of the proof of Theorem 1 implies the following Bernstein-Markov type inequality, which had previously appeared in [FHKL16].

Theorem 4. Fix $n, d \in \mathbb{N}$ with $d \in\{1, \ldots, n\}$ and let $\left(X,\|\cdot\|_{X}\right)$ be a Banach space. For every $p \in[1, \infty]$ and every function $f:\{-1,1\}^{n} \rightarrow X$ of degree at most $d$, we have

$$
\|\Delta f\|_{L_{p}\left(\{-1,1\}^{n} ; X\right)} \leqslant d^{2}\|f\|_{L_{p}\left(\{-1,1\}^{n} ; X\right)} .
$$

In [FHKL16, Lemma 5.4], the Bernstein-Markov type inequality (12) was stated only for real valued functions and $p=1$. In the vector valued setting which is of interest here, inequality (12) is sharp for general Banach spaces. Recall that a Banach space $\left(X,\|\cdot\|_{X}\right)$ has cotype $q \in[2, \infty]$ with constant $C \in(0, \infty)$ if for every $n \in \mathbb{N}$ and vectors $x_{1}, \ldots, x_{n} \in X$, we have

$$
\left(\frac{1}{2^{n}} \sum_{\varepsilon \in\{-1,1\}^{n}}\left\|\sum_{i=1}^{n} \varepsilon_{i} x_{i}\right\|_{X}^{q}\right)^{1 / q} \geqslant \frac{1}{C}\left(\sum_{i=1}^{n}\left\|x_{i}\right\|_{X}^{q}\right)^{1 / q}
$$


The fact that every Banach space $\left(X,\|\cdot\|_{X}\right)$ has cotype $q=\infty$ with constant $C=1$ follows from the triangle inequality, yet having finite cotype is a meaningful structural property of a given space. We refer to the survey [Mau03] for further information on the rich theory of type and cotype. We will show that any improvement of (12), forces the target space $X$ to have finite cotype.

Theorem 5. Let $\left(X,\|\cdot\|_{X}\right)$ be a Banach space and $p \in[1, \infty]$. Suppose that there exist some $\eta \in(0,1)$ and $d \in \mathbb{N}$ such that for every $n \in \mathbb{N}$ with $d \in\{1, \ldots, n\}$, every function $f:\{-1,1\}^{n} \rightarrow X$ of degree at most $d$ satisfies the inequality

$$
\|\Delta f\|_{L_{p}\left(\{-1,1\}^{n} ; X\right)} \leqslant(1-\eta) d^{2}\|f\|_{L_{p}\left(\{-1,1\}^{n} ; X\right)} .
$$

Then $X$ has finite cotype.

1.2. Estimates for $K$-convex Banach spaces. Let $\left(X,\|\cdot\|_{X}\right)$ be a Banach space. The $X$-valued Rademacher projection Rad : $L_{p}\left(\{-1,1\}^{n} ; X\right) \rightarrow L_{p}\left(\{-1,1\}^{n} ; X\right)$ is the operator given by

$$
\forall \varepsilon \in\{-1,1\}^{n}, \quad \operatorname{Rad}(f)(\varepsilon) \stackrel{\text { def }}{=} \sum_{i=1}^{n} \widehat{f}(\{i\}) \varepsilon_{i} .
$$

We say that $\left(X,\|\cdot\|_{X}\right)$ is $K$-convex (see also $\left[\right.$ Mau03]) if $\sup _{n \in \mathbb{N}}\|\operatorname{Rad}\|_{L_{p}\left(\{-1,1\}^{n} ; X\right) \rightarrow L_{p}\left(\{-1,1\}^{n} ; X\right)}<$ $\infty$ for some (equivalently, for all) $p \in(1, \infty)$. Pisier's $K$-convexity theorem [Pis82] asserts that a Banach space $\left(X,\|\cdot\|_{X}\right)$ is $K$-convex if and only if $X$ does not contain copies of $\left\{\ell_{1}^{n}\right\}_{n=1}^{\infty}$ with distortion arbitrarily close to 1 . Moreover, both conditions are equivalent to $X$ having nontrivial type, see [Pis73]. In the proofs of most results of this section, we will crucially use the deep fact that the heat semigroup with values in a $K$-convex space is a bounded analytic semigroup [Pis82].

1.2.1. The heat smoothing conjecture. In [MN14], Mendel and Naor asked whether for every $K$ convex Banach space $\left(X,\|\cdot\|_{X}\right)$ and $p \in(1, \infty)$ there exist $c(p, X), C(p, X) \in(0, \infty)$ such that for every $n \in \mathbb{N}$, every function $f:\{-1,1\}^{n} \rightarrow X$ in the $d$-th tail space satisfies the estimate

$$
\forall t \geqslant 0, \quad\left\|e^{-t \Delta} f\right\|_{L_{p}\left(\{-1,1\}^{n} ; X\right)} \leqslant C(p, X) e^{-c(p, X) d t}\|f\|_{L_{p}\left(\{-1,1\}^{n} ; X\right)} .
$$

In the direction of this question, currently known as the heat smoothing conjecture, they showed the following theorem, partially relying on ideas from [Pis07] (see also the work [HMO17] of Heilman, Mossel and Oleszkiewicz for an optimal result when $d=1$ and $X=\mathbb{C}$ ).

Theorem 6 (Mendel-Naor). Let $\left(X,\|\cdot\|_{X}\right)$ be a K-convex Banach space. For every $p \in(1, \infty)$, there exist $c(p, X), C(p, X) \in(0, \infty)$ and $A(p, X) \in[1, \infty)$ such that for every $n, d \in \mathbb{N}$ with $d \in\{0,1, \ldots, n-1\}$ and every function $f:\{-1,1\}^{n} \rightarrow X$ in the $d$-th tail space, we have

$$
\forall t \geqslant 0, \quad\left\|e^{-t \Delta} f\right\|_{L_{p}\left(\{-1,1\}^{n} ; X\right)} \leqslant C(p, X) e^{-c(p, X) d \min \left\{t, t^{A(p, X)}\right\}}\|f\|_{L_{p}\left(\{-1,1\}^{n} ; X\right)} .
$$

In Section 3 below, we present a simple proof of Theorem 6 relying on a duality argument.

1.2.2. An improved lower bound on the decay of the heat semigroup. Under the assumption that the target space $\left(X,\|\cdot\|_{X}\right)$ is $K$-convex, we get the following improvement over Theorem 1 (see also equation (45) in Remark 18 for comparison).

Theorem 7. Let $\left(X,\|\cdot\|_{X}\right)$ be a $K$-convex Banach space. For every $p \in(1, \infty)$, there exist $c(p, X), C(p, X) \in(0, \infty)$ and $\eta(p, X) \in\left(\frac{1}{2}, 1\right]$ such that for every $n, d \in \mathbb{N}$ with $d \in\{1, \ldots, n\}$ and every function $f:\{-1,1\}^{n} \rightarrow X$ of degree at most $d$, we have

$$
\forall t \geqslant 0, \quad\left\|e^{-t \Delta} f\right\|_{L_{p}\left(\{-1,1\}^{n} ; X\right)} \geqslant c(p, X) e^{-C(p, X) d \max \left\{t, t^{\eta(p, X)}\right\}}\|f\|_{L_{p}\left(\{-1,1\}^{n} ; X\right)} .
$$


Theorem 7 should be understood as the dual of Mendel and Naor's bound (17) for the heat smoothing conjecture. We conjecture that one can in fact take $\eta(p, X)=1$ in Theorem 7 for every $p \in(1, \infty)$ and $K$-convex Banach space $\left(X,\|\cdot\|_{X}\right)$, but a proof of such a claim appears intractable with the technique presented here. A real valued version of this conjecture for $p=1$ has previously appeared in $\left[\mathrm{FHH}^{+} 14\right.$, Section 5]. As in the case of Theorem 1, Theorem 7 also implies moment comparison for functions of low degree. We postpone the relevant result (Corollary 31) to Section 3.

1.2.3. Bernstein-Markov type inequalities for the hypercube Laplacian and the gradient. Under the additional assumption that the target space $\left(X,\|\cdot\|_{X}\right)$ is $K$-convex, we can obtain the following asymptotic improvement of the bound of Theorem 4 .

Theorem 8. Let $\left(X,\|\cdot\|_{X}\right)$ be a $K$-convex Banach space. For every $p \in(1, \infty)$, there exist $\alpha(p, X) \in[1,2)$ and $C(p, X) \in(0, \infty)$ such that for every $n, d \in \mathbb{N}$ with $d \in\{1, \ldots, n\}$ and every function $f:\{-1,1\}^{n} \rightarrow X$ of degree at most $d$, we have

$$
\|\Delta f\|_{L_{p}\left(\{-1,1\}^{n} ; X\right)} \leqslant C(p, X) d^{\alpha(p, X)}\|f\|_{L_{p}\left(\{-1,1\}^{n} ; X\right)} .
$$

We conjecture that the conclusion of Theorem 8 holds true with $\alpha(p, X)=1$ for every $p \in(1, \infty)$ and $K$-convex Banach space $\left(X,\|\cdot\|_{X}\right)$ (see also Remark 33 below). Since every $K$-convex Banach space has finite cotype, the conclusion of Theorem 8 is consistent with Theorem 5 . However, there exist Banach spaces (e.g. $X=\ell_{1}$ ) which have finite cotype but are not $K$-convex. It remains an interesting (and potentially challenging) open problem to understand whether the dependence on the degree in the vector valued Bernstein-Markov inequality (12) (even for, say, $p=2$ ) can be improved to $o\left(d^{2}\right)$ under the minimal assumption that $\left(X,\|\cdot\|_{X}\right)$ has finite cotype.

We conclude this section by presenting a Bernstein-Markov type inequality where the hypercube Laplacian is replaced by the vector valued gradient appearing in Pisier's inequality (10).

Theorem 9. Let $\left(X,\|\cdot\|_{X}\right)$ be a $K$-convex Banach space. For every $p \in(1, \infty)$ there exist $\alpha(p, X) \in[1,2)$ and $C(p, X) \in(0, \infty)$ such that for every $n, d \in \mathbb{N}$ with $d \in\{1, \ldots, n\}$ and every function $f:\{-1,1\}^{n} \rightarrow X$ of degree at most $d$, we have

$$
\left(\frac{1}{2^{n}} \sum_{\delta \in\{-1,1\}^{n}}\left\|\sum_{i=1}^{n} \delta_{i} \partial_{i} f\right\|_{L_{p}\left(\{-1,1\}^{n} ; X\right)}^{p}\right)^{1 / p} \leqslant C(p, X) d^{\alpha(p, X)}(\log d+1)\|f\|_{L_{p}\left(\{-1,1\}^{n} ; X\right)} .
$$

1.2.4. A reverse Bernstein-Markov inequality for $\Delta$. In [MN14], Mendel and Naor asked if for every $K$-convex Banach space $\left(X,\|\cdot\|_{X}\right)$ and $p \in(1, \infty)$ there exists $c(p, X) \in(0, \infty)$ such that for every $n \in \mathbb{N}$ and $d \in\{0,1, \ldots, n-1\}$, every function $f:\{-1,1\}^{n} \rightarrow X$ in the $d$-th tail space satisfies

$$
\|\Delta f\|_{L_{p}\left(\{-1,1\}^{n} ; X\right)} \geqslant c(p, X) d\|f\|_{L_{p}\left(\{-1,1\}^{n} ; X\right)} .
$$

Similar estimates for scalar valued functions had also been obtained by Meyer in [Mey84]. The following theorem, whose proof relies on a recent inequality of Erdélyi, contains a result in this direction under the additional assumption that the spectrum of the function $f$ is also bounded above.

Theorem 10. Let $\left(X,\|\cdot\|_{X}\right)$ be a $K$-convex Banach space. For every $p \in(1, \infty)$ there exists $c(p, X) \in(0, \infty)$ such that for every $n, d, m \in \mathbb{N}$ with $d+m \leqslant n$ and every function $f:\{-1,1\}^{n} \rightarrow X$ of degree at most $d+m$ which is also in the $d$-th tail space, we have

$$
\|\Delta f\|_{L_{p}\left(\{-1,1\}^{n} ; X\right)} \geqslant c(p, X) \frac{d}{m}\|f\|_{L_{p}\left(\{-1,1\}^{n} ; X\right)} .
$$

In particular, Theorem 10 provides a positive answer to the question of [MN14] in the special case when $m=O(1)$ and improves upon the previously known bounds of Meyer [Mey84] and Mendel and Naor [MN14] when $m$ is a small enough power of $d$. 
1.3. Estimates for scalar valued functions. In this section we will present explicit estimates which hold true for scalar valued functions. Even though several of the following results are special cases of theorems from the previous section, we present the full statements for the convenience of the reader not interested in the general vector valued setting.

1.3.1. The decay of the heat semigroup. The optimal bounds that can be derived from our approach for the action of the heat semigroup on functions with bounded spectrum are the following.

Theorem 11. For $p \in[1, \infty]$, let $\theta_{p}=2 \arcsin \left(\frac{2 \sqrt{p-1}}{p}\right)$. Then, for every $p \in(1, \infty), n, d \in \mathbb{N}$ with $d \in\{0,1, \ldots, n\}$ and every function $f:\{-1,1\}^{n} \rightarrow \mathbb{C}$ of degree at most $d$, we have

$$
\forall t \geqslant 0, \quad\left\|e^{-t \Delta} f\right\|_{L_{p}\left(\{-1,1\}^{n} ; \mathbb{C}\right)} \geqslant\left(\frac{\left(e^{t}+1\right)^{\frac{\pi}{2 \pi-\theta_{p}}}-\left(e^{t}-1\right)^{\frac{\pi}{2 \pi-\theta_{p}}}}{\left(e^{t}+1\right)^{\frac{\pi}{2 \pi-\theta_{p}}}+\left(e^{t}-1\right)^{\frac{\pi}{2 \pi-\theta_{p}}}}\right)^{d}\|f\|_{L_{p}\left(\{-1,1\}^{n} ; \mathbb{C}\right) .} .
$$

Moreover, for every function $f:\{-1,1\}^{n} \rightarrow \mathbb{C}$ in the $d$-th tail space, we have

$$
\forall t \geqslant 0, \quad\left\|e^{-t \Delta} f\right\|_{L_{p}\left(\{-1,1\}^{n} ; \mathbb{C}\right)} \leqslant\left(\frac{\left(1+e^{-t}\right)^{\frac{\pi}{\theta_{p}}}-\left(1-e^{-t}\right)^{\frac{\pi}{\theta_{p}}}}{\left(1+e^{-t}\right)^{\frac{\pi}{\theta_{p}}}+\left(1-e^{-t}\right)^{\frac{\pi}{\theta_{p}}}}\right)^{d}\|f\|_{L_{p}\left(\{-1,1\}^{n} ; \mathbb{C}\right)} .
$$

The above lower bound (23) on the decay of the heat semigroup combined with classical hypercontractivite estimates [Bon70] implies the following improved moment comparison result for functions of low degree.

Corollary 12. For $p \in[1, \infty]$, let $\theta_{p}=2 \arcsin \left(\frac{2 \sqrt{p-1}}{p}\right)$. Then, for every $p>q>1$, every $n, d \in \mathbb{N}$ with $d \in\{1, \ldots, n\}$ and every function $f:\{-1,1\}^{n} \rightarrow \mathbb{C}$ of degree at most $d$, we have

$$
\|f\|_{L_{p}\left(\{-1,1\}^{n} ; \mathbb{C}\right)} \leqslant\left(\frac{(\sqrt{p-1}+\sqrt{q-1})^{\frac{\pi}{2 \pi-\theta_{p}}}+(\sqrt{p-1}-\sqrt{q-1})^{\frac{\pi}{2 \pi-\theta_{p}}}}{(\sqrt{p-1}+\sqrt{q-1})^{\frac{\pi}{2 \pi-\theta_{p}}}-(\sqrt{p-1}-\sqrt{q-1})^{\frac{\pi}{2 \pi-\theta_{p}}}}\right)^{d}\|f\|_{L_{q}\left(\{-1,1\}^{n} ; \mathbb{C}\right)} .
$$

Even though Bonami's hypercontractive estimates [Bon70] break down at the endpoint $p=1$, a well known trick (see [O'D14, Theorem 9.22]) implies that if $f:\{-1,1\}^{n} \rightarrow \mathbb{C}$ has degree at most $d$,

$$
\|f\|_{L_{2}\left(\{-1,1\}^{n} ; \mathbb{C}\right)} \leqslant e^{d}\|f\|_{L_{1}\left(\{-1,1\}^{n} ; \mathbb{C}\right)} .
$$

Relying on works of Beckner [Bec75] and Weissler [Wei79], we prove the following improved bound.

Theorem 13. For every $n, d \in \mathbb{N}$ with $d \in\{1, \ldots, n\}$ and every function $f:\{-1,1\}^{n} \rightarrow \mathbb{C}$ of degree at most $d$, we have

$$
\|f\|_{L_{2}\left(\{-1,1\}^{n} ; \mathbb{C}\right)} \leqslant(2.69076)^{d}\|f\|_{L_{1}\left(\{-1,1\}^{n} ; \mathbb{C}\right)} .
$$

1.3.2. Bernstein-Markov type inequalities. The bounds (19) in the Bernstein-Markov inequality for the hypercube Laplacian take the following explicit form for scalar valued functions.

Theorem 14. For $p \in[1, \infty]$, let $\theta_{p}=2 \arcsin \left(\frac{2 \sqrt{p-1}}{p}\right)$. Then, for every $n, d \in \mathbb{N}$ with $d \in$ $\{1, \ldots, n\}$ and every function $f:\{-1,1\}^{n} \rightarrow \mathbb{C}$ of degree at most $d$, we have

$$
\|\Delta f\|_{L_{p}\left(\{-1,1\}^{n} ; \mathbb{C}\right)} \leqslant 10 d^{2-\frac{\theta_{p}}{\pi}}\|f\|_{L_{p}\left(\{-1,1\}^{n} ; \mathbb{C}\right)} .
$$

For $p \in[1, \infty]$ and a function $f:\{-1,1\}^{n} \rightarrow \mathbb{C}$, denote by

$$
\|\nabla f\|_{L_{p}\left(\{-1,1\}^{n} ; \mathbb{C}\right)} \stackrel{\text { def }}{=}\left\|\left(\sum_{i=1}^{n}\left(\partial_{i} f\right)^{2}\right)^{1 / 2}\right\|_{L_{p}\left(\{-1,1\}^{n} ; \mathbb{C}\right)} .
$$

In contrast to the vector valued Theorem 9, we can prove the following improved Bernstein-Markov type inequality for the gradient of scalar valued functions as a consequence of Theorem 14 . 
Theorem 15. For $p \in[1, \infty]$, let $\theta_{p}=2 \arcsin \left(\frac{2 \sqrt{p-1}}{p}\right)$. Then, for every $p \in(1, \infty)$, there exists $C_{p} \in(0, \infty)$ such that for every $n, d \in \mathbb{N}$ with $d \in\{1, \ldots, n\}$ and every function $f:\{-1,1\}^{n} \rightarrow \mathbb{C}$ of degree at most $d$, we have

$$
\|\nabla f\|_{L_{p}\left(\{-1,1\}^{n} ; \mathbb{C}\right)} \leqslant\left\{\begin{array}{ll}
C_{p} d^{\frac{2}{p}-\frac{\theta_{p}}{p \pi}} \log (d+1)\|f\|_{L_{p}\left(\{-1,1\}^{n} ; \mathbb{C}\right)}, & \text { if } p \in(1,2) \\
C_{p} d^{1-\frac{\theta_{p}}{2 \pi}}\|f\|_{L_{p}\left(\{-1,1\}^{n} ; \mathbb{C}\right)}, & \text { if } p \in[2, \infty)
\end{array} .\right.
$$

The change in the exponent from the range $p \in(1,2)$ to the range $p \in[2, \infty)$ and its relation to discrete Riesz transforms is further discussed in Section 4. We also postpone until then the statement of some endpoint $(p=\infty$ and $p=1)$ Bernstein-Markov inequalities for the discrete gradient (see Proposition 46 and Remark 49 respectively).

We finally turn to a problem studied by Filmus, Hatami, Keller and Lifshitz in [FHKL16] (see also [BB14]). Following their notation, for $p \in[1, \infty)$ and a function $f:\{-1,1\}^{n} \rightarrow \mathbb{C}$, denote by

$$
\operatorname{Inf}^{(p)} f \stackrel{\text { def }}{=} \sum_{i=1}^{n}\left\|\partial_{i} f\right\|_{L_{p}\left(\{-1,1\}^{n} ; \mathbb{C}\right)}^{p} .
$$

Motivated by a question of Aaronson and Ambrainis [AA14], the authors were interested in obtaining bounds of the form

$$
\operatorname{Inf}^{(p)} f \leqslant f_{p}(d)\|f\|_{L_{\infty}\left(\{-1,1\}^{n} ; \mathbb{C}\right)}^{p},
$$

where $f_{p}(d)$ is a polynomial in $d$. In this direction, they proved (32) with $f_{p}(d)=d^{3-p}$ when $p \in[1,2]$ and $f_{p}(d)=d$ when $p \in[2, \infty)$, the latter of which is sharp. Using Theorem 11, we deduce the following improved bounds for $p \in\left(1, \frac{4}{3}\right)$.

Corollary 16. For every $p \in\left(1, \frac{4}{3}\right)$ there exists a constant $K_{p} \in(0, \infty)$ such that for every $n, d \in \mathbb{N}$ with $d \in\{1, \ldots, n\}$ and every function $f:\{-1,1\}^{n} \rightarrow \mathbb{C}$ of degree at most $d$, we have

$$
\operatorname{Inf}^{(p)} f \leqslant K_{p} d^{2-\frac{1}{\pi} \arcsin \left(\frac{2 \sqrt{p-1}}{p}\right)}\|f\|_{L_{\infty}\left(\{-1,1\}^{n} ; \mathbb{C}\right)}^{p} .
$$

1.3.3. A reverse Bernstein-Markov type inequality for $\nabla$. For the case of scalar valued functions we will also prove the following variant of Theorem 10 for the discrete gradient.

Theorem 17. For every $p \in(1, \infty)$ there exists $c_{p} \in(0, \infty)$ such that for every $n, d, m \in \mathbb{N}$ with $d+m \leqslant n$ and every function $f:\{-1,1\}^{n} \rightarrow \mathbb{C}$ of degree at most $d+m$ which is also in the $d$-th tail space, we have

$$
\|\nabla f\|_{L_{p}\left(\{-1,1\}^{n} ; \mathbb{C}\right)} \geqslant c_{p} \sqrt{\frac{d}{m}}\|f\|_{L_{p}\left(\{-1,1\}^{n} ; \mathbb{C}\right)}
$$

Acknowledgements. We are indebted to Assaf Naor for many helpful discussions. We are also very grateful to Tamás Erdélyi for proving the main result of [Erd20] upon our request and to an anonymous referee for sharing with us an argument which improved Corollary 21. Finally, we would like to thank Franoise Lust-Piquard for valuable feedback.

\section{Estimates for a general Banach SPACE}

We first present the proof of Theorem 1, the lower bound on the decay of the heat semigroup acting on functions with values in a general Banach space. Recall that the $d$-th Chebyshev polynomial of the first kind $T_{d}(x)$ is the unique polynomial of degree $d$ such that $T_{d}(\cos \theta)=\cos (d \theta)$ for every $\theta \in \mathbb{R}$. Chebyshev's inequality [BE95, p. 235] asserts that the $d$-th Chebyshev polynomial of the first kind is characterized by the extremal property

$$
\forall x \in \mathbb{R} \backslash[-1,1], \quad\left|T_{d}(x)\right|=\max \left\{|p(x)|: \operatorname{deg}(p) \leqslant d \text { and }\|p\|_{\mathcal{e}([-1,1])}=1\right\},
$$


where for a continuous function $h: K \rightarrow \mathbb{C}$ on a compact space $K$, we set $\|h\|_{\mathcal{e}(K)}=\max _{x \in K}|h(x)|$. Proof of Theorem 1. Fix $n \in \mathbb{N}, d \in\{1, \ldots, n\}, p \in[1, \infty]$ and let $f:\{-1,1\}^{n} \rightarrow X$ be a function of degree at most $d$. The contractivity of the heat semigroup which we derived from (7), can be rewritten as $\left\|x^{\Delta} f\right\|_{L_{p}\left(\{-1,1\}^{n} ; X\right)} \leqslant\|f\|_{L_{p}\left(\{-1,1\}^{n} ; X\right)}$ for every $x \in[0,1]$. However, for $x \in \mathbb{R}$,

$$
(-x)^{\Delta} f(\varepsilon)=\sum_{A \subseteq\{1, \ldots, n\}}(-x)^{|A|} \widehat{f}(A) w_{A}(\varepsilon)=\sum_{A \subseteq\{1, \ldots, n\}} x^{|A|} \widehat{f}(A) w_{A}(-\varepsilon)=\left(x^{\Delta} f\right)(-\varepsilon),
$$

thus $\left\|(-x)^{\Delta} f\right\|_{L_{p}\left(\{-1,1\}^{n} ; X\right)}=\left\|x^{\Delta} f\right\|_{L_{p}\left(\{-1,1\}^{n} ; X\right)}$. Consequently,

$$
\forall x \in[-1,1], \quad\left\|x^{\Delta} f\right\|_{L_{p}\left(\{-1,1\}^{n} ; X\right)} \leqslant\|f\|_{L_{p}\left(\{-1,1\}^{n} ; X\right)} .
$$

Let $\mu$ be any complex measure on $[-1,1]$. Then, averaging over $(37)$, we get

$$
\begin{aligned}
\| \int_{-1}^{1} x^{\Delta} f \mathrm{~d} \mu(x) & \left\|_{L_{p}\left(\{-1,1\}^{n} ; X\right)} \leqslant \int_{-1}^{1}\right\| x^{\Delta} f \|_{L_{p}\left(\{-1,1\}^{n} ; X\right)} \mathrm{d}|\mu|(x) \\
& \stackrel{(37)}{\leqslant} \int_{-1}^{1}\|f\|_{L_{p}\left(\{-1,1\}^{n} ; X\right)} \mathrm{d}|\mu|(x)=\|\mu\|_{\mathcal{M}([-1,1])}\|f\|_{L_{p}\left(\{-1,1\}^{n} ; X\right)},
\end{aligned}
$$

where for a complex measure $\mu$ on a compact space $K$, we denote by $\|\mu\|_{\mathcal{M}(K)}$ the total variation of $\mu$. For $t \geqslant 0$, consider the linear functional $\varphi_{t}:\left(\operatorname{span}\left\{1, x, \ldots, x^{d}\right\},\|\cdot\|_{e}([-1,1])\right) \rightarrow \mathbb{C}$ given by

$$
\varphi_{t}\left(\sum_{k=0}^{d} a_{k} x^{k}\right) \stackrel{\text { def }}{=} \sum_{k=0}^{d} a_{k} e^{t k}
$$

or $\varphi_{t}(p)=p\left(e^{t}\right)$ when $p$ is a polynomial with $\operatorname{deg}(p) \leqslant d$. Then, Chebyshev's inequality (35) can be rewritten as

$$
\forall p \in \operatorname{span}\left\{1, x, \ldots, x^{d}\right\}, \quad\left|\varphi_{t}(p)\right| \leqslant T_{d}\left(e^{t}\right)\|p\|_{\mathcal{e}([-1,1])}
$$

Therefore, by the Hahn-Banach theorem and the Riesz representation theorem, there exists a complex measure $\mu_{t}$ on $[-1,1]$ such that $\left\|\mu_{t}\right\|_{\mathcal{M}([-1,1])} \leqslant T_{d}\left(e^{t}\right)$ and for every polynomial $p$, we have

$$
\operatorname{deg}(p) \leqslant d \quad \Longrightarrow \quad \int_{-1}^{1} p(x) \mathrm{d} \mu_{t}(x)=p\left(e^{t}\right)
$$

Since $\widehat{f}(A)=0$ when $|A|>d$, applying (38) for the measure $\mu_{t}$ satisfying (41), we deduce that

$$
\forall t \geqslant 0, \quad\left\|e^{t \Delta} f\right\|_{L_{p}\left(\{-1,1\}^{n} ; X\right)} \stackrel{(41)}{=}\left\|\int_{-1}^{1} x^{\Delta} f \mathrm{~d} \mu_{t}(x)\right\|_{L_{p}\left(\{-1,1\}^{n} ; X\right)} \stackrel{(40)}{\leqslant} T_{d}\left(e^{t}\right)\|f\|_{L_{p}\left(\{-1,1\}^{n} ; X\right)},
$$

which is equivalent to the desired inequality (8).

Remark 18. A weaker lower bound for the decay of the heat semigroup, attributed partially to Oleszkiewicz, was established in [FHKL16, Lemma 5.4] and asserts that for every function $f$ : $\{-1,1\}^{n} \rightarrow X$ of degree at most $d$, we have

$$
\left\|e^{-t \Delta} f\right\|_{L_{p}\left(\{-1,1\}^{n} ; X\right)} \geqslant e^{-t d^{2}}\|f\|_{L_{p}\left(\{-1,1\}^{n} ; X\right)} .
$$

Inequality (43) was stated in [FHKL16] only for $X=\mathbb{R}$ and $p=1$, but the argument presented there works in greater generality. Checking that (43) is weaker than the estimate (8) amounts to showing that for $y \geqslant 1$, we have $T_{d}(y) \leqslant y^{d^{2}}$, which can be easily derived from the identity

$$
\forall|y| \geqslant 1, \quad T_{d}(y)=\frac{\left(y+\sqrt{y^{2}-1}\right)^{d}+\left(y-\sqrt{y^{2}-1}\right)^{d}}{2} .
$$

Furthermore, using (44), it is elementary to check that

$$
\forall t \geqslant 0, \quad T_{d}\left(e^{t}\right) \leqslant e^{3 \max \{t, \sqrt{t}\} d},
$$


therefore inequality (18) is also an improvement over $(8)$ for $t \approx 0$.

Proof of Corollary 2. Fix $n \in \mathbb{N}, d \in\{1, \ldots, n\}, p>q>1$ and a function $f:\{-1,1\}^{n} \rightarrow X$ of degree at most $d$. Bonami's hypercontractive inequality [Bon70] (the straightforward vector valued extension of which is due to Borell, see [Bor79]) asserts that

$$
\forall t \geqslant \frac{1}{2} \log \left(\frac{p-1}{q-1}\right), \quad\left\|e^{-t \Delta} f\right\|_{L_{p}\left(\{-1,1\}^{n} ; X\right)} \leqslant\|f\|_{L_{q}\left(\{-1,1\}^{n} ; X\right)} .
$$

Combining (8) and (46), we deduce that for $t \geqslant \frac{1}{2} \log \left(\frac{p-1}{q-1}\right)$,

$$
\|f\|_{L_{p}\left(\{-1,1\}^{n} ; X\right)} \stackrel{(8)}{\leqslant} T_{d}\left(e^{t}\right)\left\|e^{-t \Delta} f\right\|_{L_{p}\left(\{-1,1\}^{n} ; X\right)} \stackrel{(46)}{\leqslant} T_{d}\left(e^{t}\right)\|f\|_{L_{q}\left(\{-1,1\}^{n} ; X\right)} .
$$

Plugging $t=\frac{1}{2} \log \left(\frac{p-1}{q-1}\right)$ in (47), we deduce the moment comparison (9).

Remark 19. Inequality (9) for $d=1$ with some constant $C(p, q)$ originated in the work [Kah64] of Kahane and the implicit constant was later improved to $\sqrt{\frac{p-1}{q-1}}$ by Kwapień in [Kwa76]. The use of hypercontractivity for moment comparison of vector valued Walsh polynomials was initiated by Borell in [Bor79] (see the exposition [Pis78]), who later showed in [Bor84] that inequality (9) holds true with some constant depending only on $p, q$ and $d$ (this had previously been proven for scalar valued functions by Bourgain in [Bou80] via a square function approach). To the extent of our knowledge, the best known dependence in (9) before the present work could be extracted from an argument in the monograph [KW92, Proposition 6.5.1] which goes as follows. For $k \in\{0,1, \ldots, n\}$ let $\operatorname{Rad}_{k}: L_{p}\left(\{-1,1\}^{n} ; X\right) \rightarrow L_{p}\left(\{-1,1\}^{n} ; X\right)$ be the Rademacher projection on level $k$ given by

$$
\forall \varepsilon \in\{-1,1\}^{n}, \quad \operatorname{Rad}_{k}(f)(\varepsilon) \stackrel{\text { def }}{=} \sum_{\substack{A \subseteq\{1, \ldots, n\} \\|A|=k}} \widehat{f}(A) w_{A}(\varepsilon) .
$$

If $d \in\{0,1, \ldots, n\}$, consider the linear functional $\sigma_{k}:\left(\operatorname{span}\left\{1, x, \ldots, x^{d}\right\},\|\cdot\| \mathfrak{e}([-1,1])\right) \rightarrow \mathbb{C}$ given by $\sigma_{k}(p)=\frac{p^{(k)}(0)}{k !}$. Then, for every $k \in\{1, \ldots, d\}$, there exists some constant $c(d, k) \in(0, \infty)$ such that

$$
\operatorname{deg}(p) \leqslant d \Longrightarrow\left|\sigma_{k}(p)\right| \leqslant c(d, k)\|p\|_{\mathcal{e}([-1,1])} .
$$

The optimal value of $c(d, k)$ is known, see [BE95, p. 248], but clearly $c(d, k) \geqslant \frac{\left|T_{d}^{(k)}(0)\right|}{k !}$. Repeating the duality argument used in the proof of Theorem 1, we deduce that for every function $f$ : $\{-1,1\}^{n} \rightarrow X$ of degree at most $d, q \in[1, \infty]$ and $k \in\{0, \ldots, d\}$,

$$
\left\|\operatorname{Rad}_{k} f\right\|_{L_{q}\left(\{-1,1\}^{n} ; X\right)} \leqslant c(d, k)\|f\|_{L_{q}\left(\{-1,1\}^{n} ; X\right)} .
$$

Furthermore, by the moment comparison of homogeneous Walsh polynomials [Bor79], for $p>q>1$,

$$
\left\|\operatorname{Rad}_{k} f\right\|_{L_{p}\left(\{-1,1\}^{n} ; X\right)} \leqslant\left(\frac{p-1}{q-1}\right)^{k / 2}\left\|\operatorname{Rad}_{k} f\right\|_{L_{q}\left(\{-1,1\}^{n} ; X\right)} .
$$

Therefore, combining (50) and (51), we get

$$
\begin{aligned}
\|f\|_{L_{p}\left(\{-1,1\}^{n} ; X\right)} \leqslant \sum_{k=0}^{d}\left\|\operatorname{Rad}_{k} f\right\|_{L_{p}\left(\{-1,1\}^{n} ; X\right)} \stackrel{(51)}{\leqslant} \sum_{k=0}^{d}\left(\frac{p-1}{q-1}\right)^{k / 2}\left\|\operatorname{Rad}_{k} f\right\|_{L_{q}\left(\{-1,1\}^{n} ; X\right)} \\
\stackrel{(50)}{\leqslant} \sum_{k=0}^{d} c(d, k)\left(\frac{p-1}{q-1}\right)^{k / 2}\|f\|_{L_{q}\left(\{-1,1\}^{n} ; X\right)} .
\end{aligned}
$$


However, the constant obtained by this argument is

$$
\sum_{k=0}^{d} c(d, k)\left(\frac{p-1}{q-1}\right)^{k / 2} \geqslant \sum_{k=0}^{d} \frac{\left|T_{d}^{(k)}(0)\right|}{k !}\left(\frac{p-1}{q-1}\right)^{k / 2} \geqslant \sum_{k=0}^{d} \frac{T_{d}^{(k)}(0)}{k !}\left(\frac{p-1}{q-1}\right)^{k / 2}=T_{d}\left(\sqrt{\frac{p-1}{q-1}}\right),
$$

and therefore Corollary 2 improves over the result of [KW92].

Combining Corollary 2 and (45), it follows that for $p>q>1$ and every function $f:\{-1,1\}^{n} \rightarrow$ $X$ of degree at most $d$, we have

$$
\|f\|_{L_{p}\left(\{-1,1\}^{n} ; X\right)} \leqslant e^{3 d \max \left\{\frac{1}{2} \log \left(\frac{p-1}{q-1}\right), \frac{1}{\sqrt{2}} \log \left(\frac{p-1}{q-1}\right)^{1 / 2}\right\}}\|f\|_{L_{q}\left(\{-1,1\}^{n} ; X\right)} .
$$

In particular, if $p-1 \geqslant(1+\varepsilon)(q-1)$ for some $\varepsilon>0$, then there exists $C_{\varepsilon}=O(1 / \sqrt{\varepsilon})>0$ such that every function $f:\{-1,1\}^{n} \rightarrow X$ of degree at most $d$ satisfies

$$
\|f\|_{L_{p}\left(\{-1,1\}^{n} ; X\right)} \leqslant\left(\frac{p-1}{q-1}\right)^{C_{\varepsilon} d}\|f\|_{L_{q}\left(\{-1,1\}^{n} ; X\right)},
$$

which should be understood as an extension of Borell's moment comparison (51) for vector valued functions of low degree instead of homogeneous. The validity of Theorem 7 with $\eta(p, X)=1$ would imply the estimate (55) with $C_{\varepsilon}$ replaced by a universal contant $C \in(0, \infty)$.

Remark 20. Some similar moment comparison estimates can be obtained for functions on the $\alpha$ biased hypercube using the biased hypercontractivity of Oleszkiewicz [Ole03] and Wolff [Wol07]. Indeed, it follows from the main results of [Ole03, Wol07] and convexity considerations that for every $p>q>1$ and $\alpha \in(0,1)$, there exists a closed interval $I_{p, q}(\alpha) \subseteq[-1,1]$ with nonempty interior containing 0 such that for every $n \in \mathbb{N}$ and every coefficients $\left\{a_{S}\right\}_{S \subseteq\{1, \ldots, n\}} \subseteq X$,

$$
\sup _{x \in I_{p, q}(\alpha)}\left\|\sum_{S \subseteq\{1, \ldots, n\}} x^{|S|} a_{S} w_{S}\right\|_{L_{p}\left(\left\{-\gamma^{-1}, \gamma\right\}^{n}, \mu_{\alpha}^{n} ; X\right)} \leqslant\left\|\sum_{S \subseteq\{1, \ldots, n\}} a_{S} w_{S}\right\|_{L_{q}\left(\left\{-\gamma^{-1}, \gamma\right\}^{n}, \mu_{\alpha}^{n} ; X\right)},
$$

where $\mu_{\alpha}=\alpha \delta_{-\gamma^{-1}}+(1-\alpha) \delta_{\gamma}$ and $\gamma=\sqrt{\frac{1}{\alpha}-1}$. Running the same duality argument as in the proof of Theorem 1 combined with an application of Chebyshev's inequality (35) for a symmetric interval $J_{p, q}(\alpha) \subseteq I_{p, q}(\alpha)$, proves an analogue of $(9)$ on $\left(\left\{-\gamma^{-1}, \gamma\right\}^{n}, \mu_{\alpha}^{n}\right)$.

Finally we state one "endpoint" version ${ }^{1}$ of our moment comparison (9). Recall that for a function $h:\{-1,1\}^{n} \rightarrow[0, \infty)$, we denote its entropy by

$$
\operatorname{Ent}(h) \stackrel{\text { def }}{=} \frac{1}{2^{n}} \sum_{\varepsilon \in\{-1,1\}^{n}} h(\varepsilon) \log h(\varepsilon)-\left(\frac{1}{2^{n}} \sum_{\varepsilon \in\{-1,1\}^{n}} h(\varepsilon)\right) \cdot \log \left(\frac{1}{2^{n}} \sum_{\varepsilon \in\{-1,1\}^{n}} h(\varepsilon)\right) .
$$

Corollary 21. Fix $n, d \in \mathbb{N}$ with $d \in\{1, \ldots, n\}$ and let $\left(X,\|\cdot\|_{X}\right)$ be a Banach space. For every $q \in(0, \infty)$ there exists a constant $C_{q} \in(0, \infty)$ such that every function $f:\{-1,1\}^{n} \rightarrow X$ of degree at most d satisfies

$$
\operatorname{Ent}\left(\|f\|_{X}^{q}\right) \leqslant C_{q} d\|f\|_{L_{q}\left(\{-1,1\}^{n} ; X\right)}^{q} .
$$

Proof. By Hölder's inequality, for every $\alpha, \beta \in(0, \infty)$ and $\lambda \in(0,1)$,

$$
\|f\|_{L_{\left(\frac{\lambda}{\alpha}+\frac{1-\lambda}{\beta}\right)^{-1}}\left(\{-1,1\}^{n} ; X\right)} \leqslant\|f\|_{L_{\alpha}\left(\{-1,1\}^{n} ; X\right)}^{\lambda}\|f\|_{L_{\beta}\left(\{-1,1\}^{n} ; X\right)}^{1-\lambda} .
$$

In other words, the function $\phi:(0, \infty) \rightarrow \mathbb{R}$ given by

$$
\forall r \in(0, \infty), \quad \phi(r) \stackrel{\text { def }}{=} \log \left(\|f\|_{L_{1 / r}\left(\{-1,1\}^{n} ; X\right)}\right)
$$

\footnotetext{
${ }^{1}$ The bound (58) of Corollary 21 was pointed out to us by an anonymous referee, who improved a suboptimal $O_{q}\left(d^{2}\right)$ estimate appearing in an earlier version of this manuscript. We are grateful to them for sharing their improvement with us and for their helpful comments.
} 
is convex. Hence, the function $(0, \infty) \ni r \longmapsto \phi^{\prime}(1 / r)$ is nonincreasing, which implies that

$$
\forall p<q, \quad \phi^{\prime}\left(\frac{1}{q}\right) \cdot\left(\frac{1}{p}-\frac{1}{q}\right) \geqslant \phi\left(\frac{1}{p}\right)-\phi\left(\frac{1}{q}\right) .
$$

Writing

$$
\|f\|_{L_{\alpha}\left(\{-1,1\}^{n} ; X\right)}=\exp \left(\frac{1}{\alpha} \log \left(\frac{1}{2^{n}} \sum_{\varepsilon \in\{-1,1\}^{n}}\|f(\varepsilon)\|_{X}^{\alpha}\right)\right)
$$

one deduces that

$$
\frac{\mathrm{d}}{\mathrm{d} \alpha}\|f\|_{L_{\alpha}\left(\{-1,1\}^{n} ; X\right)}=\|f\|_{L_{\alpha}\left(\{-1,1\}^{n} ; X\right)} \cdot \frac{\operatorname{Ent}\|f\|_{X}^{\alpha}}{\alpha^{2}\|f\|_{L_{\alpha}\left(\{-1,1\}^{n} ; X\right)}^{\alpha}},
$$

which implies that

$$
\phi^{\prime}\left(\frac{1}{q}\right)=-\frac{\operatorname{Ent}\|f\|_{X}^{q}}{\|f\|_{L_{q}\left(\{-1,1\}^{n} ; X\right)}^{q}} .
$$

By the monotonicity of $r \mapsto \phi^{\prime}(1 / r)$ and (64), in order to prove (58) for every $q \in(0, \infty)$ it suffices to consider the case $q \geqslant 4$. By (64), (61) can be rewritten as

$$
\begin{aligned}
\frac{\operatorname{Ent}\|f\|_{X}^{q}}{\|f\|_{L_{q}\left(\{-1,1\}^{n} ; X\right)}^{q}} & \leqslant \frac{p q}{q-p} \log \left(\frac{\|f\|_{L_{q}\left(\{-1,1\}^{n} ; X\right)}}{\|f\|_{L_{p}\left(\{-1,1\}^{n} ; X\right)}}\right) \\
& \stackrel{(9)}{\leqslant} \frac{p q}{q-p} \log \left(T_{d}\left(\sqrt{\frac{q-1}{p-1}}\right)\right) \leqslant \frac{p q d}{2(q-p)} \log \frac{4(q-1)}{p-1}
\end{aligned}
$$

where in the last inequality we used the estimate $T_{d}(y) \leqslant(2 y)^{d}$ for $y>1$ which follows from (44). Choosing $p=q / 2$ completes the proof with $C_{q}=O(q)$ as $q \rightarrow \infty$.

Remark 22. The application (59) of Hölder's inequality can be rewritten as

$$
\forall 0<q<p<r, \quad \log \left(\frac{\|f\|_{L_{p}\left(\{-1,1\}^{n} ; X\right)}}{\|f\|_{L_{q}\left(\{-1,1\}^{n} ; X\right)}}\right) \leqslant \frac{(p-q) r}{(r-q) p} \cdot \log \left(\frac{\|f\|_{L_{r}\left(\{-1,1\}^{n} ; X\right)}}{\|f\|_{L_{q}\left(\{-1,1\}^{n} ; X\right)}}\right) .
$$

It is classical that using (66), one can improve moment comparison bounds for low degree functions (see, e.g., [IT19] for a recent application of this idea). The same applies to the moment comparison inequalities obtained in this paper. For instance, combining (9) with (66), we deduce that for every function $f:\{-1,1\}^{n} \rightarrow X$ of degree at most $d$ and every $p>q>1$,

$$
\|f\|_{L_{p}\left(\{-1,1\}^{n} ; X\right)} \leqslant \inf _{r>p} T_{d}\left(\sqrt{\frac{r-1}{q-1}}\right)^{\frac{(p-q) r}{(r-q) p}} \cdot\|f\|_{L_{q}\left(\{-1,1\}^{n} ; X\right)} .
$$

Moreover it is well known that the same trick relying on (66) can be used to prove moment comparison estimates beyond the range $p>q>1$ (see, e.g., [O'D14, Theorem 9.22] or Theorem 13 below). Since all such applications of (66) are automatic, in the sequel we will omit stating improvements such as (67) (for instance, of Corollary 12 or Corollary 31).

We proceed by proving Pisier's inequality (11) for functions of low degree. The proof is an almost mechanical adaptation of Pisier's argument from [Pis86] (see also the exposition in [Nao12]) with the exception of suitably using the lower bound (8) instead of the trivial lower bound

$$
\forall t \geqslant 0, \quad\left\|e^{-t \Delta} f\right\|_{L_{p}\left(\{-1,1\}^{n} ; X\right)} \geqslant e^{-n t}\|f\|_{L_{p}\left(\{-1,1\}^{n} ; X\right)},
$$

which holds true for every function $f:\{-1,1\}^{n} \rightarrow X$. For completeness, we present the full proof. 
Proof of Theorem 3. Fix $p \in[1, \infty)$ and let $f:\{-1,1\}^{n} \rightarrow X$ be such that $\sum_{\delta \in\{-1.1\}^{n}} f(\delta)=0$. Fix $s \geqslant 0$ and consider a normalizing functional $g_{s}^{*}$ of $e^{-s \Delta} f$ in $L_{p}\left(\{-1,1\}^{n} ; X\right)$, that is, a function $g_{s}^{*}:\{-1,1\}^{n} \rightarrow X^{*}$ such that $\left\|g_{s}^{*}\right\|_{L_{q}\left(\{-1,1\}^{n} ; X^{*}\right)}=1$, where $\frac{1}{p}+\frac{1}{q}=1$, and

$$
\left\|e^{-s \Delta} f\right\|_{L_{p}\left(\{-1,1\}^{n} ; X\right)}=\frac{1}{2^{n}} \sum_{\varepsilon \in\{-1,1\}^{n}}\left\langle g_{s}^{*}(\varepsilon), e^{-s \Delta} f(\varepsilon)\right\rangle=\frac{1}{2^{n}} \sum_{\varepsilon \in\{-1,1\}^{n}}\left\langle e^{-s \Delta} g_{s}^{*}(\varepsilon), f(\varepsilon)\right\rangle,
$$

where in the last equality we used the fact that $e^{-s \Delta}$ is self-adjoint. However, for $\varepsilon \in\{-1,1\}^{n}$,

$$
\begin{aligned}
\left\langle e^{-s \Delta} g_{s}^{*}(\varepsilon), f(\varepsilon)\right\rangle & =\frac{1}{2^{n}} \sum_{\delta \in\{-1,1\}^{n}}\left\langle e^{-s \Delta} \Delta^{-1}\left(\sum_{i=1}^{n} \delta_{i} \partial_{i}\right) g_{s}^{*}(\varepsilon), \sum_{i=1}^{n} \delta_{i} \partial_{i} f(\varepsilon)\right\rangle \\
& =\frac{1}{2^{n}} \sum_{\delta \in\{-1,1\}^{n}} \int_{s}^{\infty}\left\langle\sum_{i=1}^{n} \delta_{i} \partial_{i} e^{-t \Delta} g_{s}^{*}(\varepsilon), \sum_{i=1}^{n} \delta_{i} \partial_{i} f(\varepsilon)\right\rangle \mathrm{d} t .
\end{aligned}
$$

For $s, t \geqslant 0$, consider the fucntion $g_{s, t}^{*}:\{-1,1\}^{n} \times\{-1,1\}^{n} \rightarrow X^{*}$ given by

$$
\begin{aligned}
g_{s, t}^{*}(\varepsilon, \delta) & \stackrel{\text { def }}{=} \sum_{A \subseteq\{1, \ldots, n\}} \widehat{g}_{s}^{*}(A) \prod_{i \in A}\left(e^{-t} \varepsilon_{i}+\left(1-e^{-t}\right) \delta_{i}\right) \\
& =e^{-t \Delta} g_{s}^{*}(\varepsilon)+\left(e^{t}-1\right) \sum_{i=1}^{n} \varepsilon_{i} \delta_{i} \partial_{i} e^{-t \Delta} g_{s}^{*}(\varepsilon)+\Phi_{s, t}^{*}(\varepsilon, \delta),
\end{aligned}
$$

where $\sum_{\delta \in\{-1,1\}^{n}} \delta_{i} \Phi_{s, t}^{*}(\varepsilon, \delta)=0$ for every $\varepsilon \in\{-1,1\}^{n}$ and $i \in\{1, \ldots, n\}$. Furthermore, for every $s, t \geqslant 0$, expanding (71) in the Walsh basis, we see that

$$
g_{s, t}^{*}(\varepsilon, \delta)=\sum_{B \subseteq\{1, \ldots, n\}} e^{-t|B|}\left(1-e^{-t}\right)^{n-|B|} g_{s}^{*}\left(\sum_{i \in B} \varepsilon_{i} e_{i}+\sum_{i \in\{1, \ldots, n\} \backslash B} \delta_{i} e_{i}\right),
$$

where $\left\{e_{i}\right\}_{i=1}^{n}$ is the standard basis of $\mathbb{R}^{n}$. Therefore, for every $s, t \geqslant 0$, we have

$$
\left\|g_{s, t}^{*}\right\|_{L_{q}\left(\{-1,1\}^{n} \times\{-1,1\}^{n} ; X^{*}\right)} \leqslant \sum_{k=0}^{n}\left(\begin{array}{l}
n \\
k
\end{array}\right) e^{-t k}\left(1-e^{-t}\right)^{n-k}\left\|g_{s}^{*}\right\|_{L_{q}\left(\{-1,1\}^{n} ; X^{*}\right)}=1 .
$$

Combining (69), (70), (71) and (73) with Hölder's inequality, we deduce that

$$
\begin{aligned}
\left\|e^{-s \Delta} f\right\|_{L_{p}\left(\{-1,1\}^{n} ; X\right)} \stackrel{(70) \wedge(71)}{=} \frac{1}{4^{n}} \sum_{\varepsilon, \delta \in\{-1,1\}^{n}} \int_{s}^{\infty} \frac{1}{e^{t}-1}\left\langle g_{s, t}^{*}(\varepsilon, \delta), \sum_{i=1}^{n} \delta_{i} \partial_{i} f(\varepsilon)\right\rangle \mathrm{d} t \\
\stackrel{(73)}{\leqslant}\left(\int_{s}^{\infty} \frac{1}{e^{t}-1} \mathrm{~d} t\right)\left(\frac{1}{2^{n}} \sum_{\delta \in\{-1,1\}^{n}}\left\|\sum_{i=1}^{n} \delta_{i} \partial_{i} f\right\|_{L_{p}\left(\{-1,1\}^{n} ; X\right)}^{p}\right)^{1 / p} \\
=\log \left(\frac{e^{s}}{e^{s}-1}\right)\left(\frac{1}{2^{n}} \sum_{\delta \in\{-1,1\}^{n}}\left\|\sum_{i=1}^{n} \delta_{i} \partial_{i} f\right\|_{L_{p}\left(\{-1,1\}^{n} ; X\right)}^{p}\right)^{1 / p} .
\end{aligned}
$$

Furthermore, since $f$ has degree at most $d$, Theorem 1 implies that for $s \geqslant 0$,

$$
\frac{1}{T_{d}\left(e^{s}\right)}\|f\|_{L_{p}\left(\{-1,1\}^{n} ; X\right)} \leqslant\left\|e^{-s \Delta} f\right\|_{L_{p}\left(\{-1,1\}^{n} ; X\right)} .
$$

Combining (74) and (75), we conclude that for every $s \geqslant 0$,

$$
\|f\|_{L_{p}\left(\{-1,1\}^{n} ; X\right)} \leqslant T_{d}\left(e^{s}\right) \log \left(\frac{e^{s}}{e^{s}-1}\right)\left(\frac{1}{2^{n}} \sum_{\delta \in\{-1,1\}^{n}}\left\|\sum_{i=1}^{n} \delta_{i} \partial_{i} f\right\|_{L_{p}\left(\{-1,1\}^{n} ; X\right)}^{p}\right)^{1 / p} .
$$


As in Remark 18, using that $T_{d}\left(e^{s}\right) \leqslant e^{d^{2} s}$ for $s \geqslant 0$ and a straightforward optimization we get

$$
\min _{s \geqslant 0} T_{d}\left(e^{s}\right) \log \left(\frac{e^{s}}{e^{s}-1}\right) \leqslant \min _{s \geqslant 0} e^{d^{2} s} \log \left(\frac{e^{s}}{e^{s}-1}\right) \leqslant 3(\log d+1) .
$$

Plugging (77) in (76), we finally deduce Pisier's inequality (11).

We now prove the Bernstein-Markov type inequality (12) for the hypercube Laplacian. In the proof, we will need Markov's inequality (see [BE95, Theorem 5.1.8]) which asserts that

$$
\max \left\{\left|p^{\prime}(1)\right|: \operatorname{deg}(p) \leqslant d \text { and }\|p\|_{\mathcal{C}([-1,1])}=1\right\}=d^{2},
$$

where the equality is achieved for the $d$-th Chebyshev polynomial of the first kind $T_{d}(x)$. Our proof is similar to the one presented in [FHKL16], which also crucially relied on Markov's inequality (78).

Proof of Theorem 4. Fix $n \in \mathbb{N}, d \in\{1, \ldots, n\}, p \in[1, \infty]$ and let $f:\{-1,1\}^{n} \rightarrow X$ be a function of degree at most $d$. Consider the linear functional $\psi:\left(\operatorname{span}\left\{1, x, \ldots, x^{d}\right\},\|\cdot\|_{\mathcal{e}([-1,1])}\right) \rightarrow \mathbb{C}$ given by

$$
\psi\left(\sum_{k=0}^{d} a_{k} x^{k}\right) \stackrel{\text { def }}{=} \sum_{k=0}^{d} k a_{k},
$$

or $\psi(p)=p^{\prime}(1)$ when $p$ is a polynomial with $\operatorname{deg}(p) \leqslant d$. Then, Markov's inequality (78) can be rewritten as

$$
\forall p \in \operatorname{span}\left\{1, x, \ldots, x^{d}\right\}, \quad|\psi(p)| \leqslant d^{2}\|p\|_{\mathcal{e}([-1,1])} .
$$

Therefore, by the Hahn-Banach theorem and the Riesz representation theorem, there exists a complex measure $v$ on $[-1,1]$ such that $\|v\|_{\mathcal{M}([-1,1])} \leqslant d^{2}$ and for every polynomial $p$, we have

$$
\operatorname{deg}(p) \leqslant d \quad \Longrightarrow \quad \int_{-1}^{1} p(x) \mathrm{d} v(x)=p^{\prime}(1)
$$

Since $\widehat{f}(A)=0$ when $|A|>d$, we get that

$$
\begin{aligned}
& \|\Delta f\|_{L_{p}\left(\{-1,1\}^{n} ; X\right)} \stackrel{(81)}{=}\left\|\int_{-1}^{1} x^{\Delta} f \mathrm{~d} v(x)\right\|_{L_{p}\left(\{-1,1\}^{n} ; X\right)} \leqslant \int_{-1}^{1}\left\|x^{\Delta} f\right\|_{L_{p}\left(\{-1,1\}^{n} ; X\right)} \mathrm{d}|v|(x) \\
& \stackrel{(37)}{\leqslant} \int_{-1}^{1}\|f\|_{L_{p}\left(\{-1,1\}^{n} ; X\right)} \mathrm{d}|v|(x)=\|v\|_{\mathcal{M}([-1,1])}\|f\|_{L_{p}\left(\{-1,1\}^{n} ; X\right)} \stackrel{(80)}{\leqslant} d^{2}\|f\|_{L_{p}\left(\{-1,1\}^{n} ; X\right)},
\end{aligned}
$$

which concludes the proof of the theorem.

Remark 23. One can generalize Theorem 4 by showing that for every $k \in \mathbb{N}$, we have

$$
\|\Delta(\Delta-1) \cdots(\Delta-k+1) f\|_{L_{p}\left(\{-1,1\}^{n} ; X\right)} \leqslant \frac{d^{2}\left(d^{2}-1^{2}\right) \cdots\left(d^{2}-(k-1)^{2}\right)}{1 \cdot 3 \cdots(2 k-1)}\|f\|_{L_{p}\left(\{-1,1\}^{n} ; X\right)} .
$$

Theorem 1 corresponds to the case $k=1$. To derive (83), one can use the same duality argument used in the proofs of Theorems 1 and 4 for the linear functional $\psi_{k}(p)=p^{(k)}(1)$ along with Markov's inequality for higher derivatives (see [BE95, Theorem 5.2.1]), which asserts that

$$
\max \left\{\left|p^{(k)}(1)\right|: \operatorname{deg}(p) \leqslant d \text { and }\|p\|_{\mathcal{e}([-1,1])}=1\right\}=T_{d}^{(k)}(1)=\frac{d^{2}\left(d^{2}-1^{2}\right) \cdots\left(d^{2}-(k-1)^{2}\right)}{1 \cdot 3 \cdots(2 k-1)} .
$$

We conclude this section by proving Theorem 5 .

Proof of Theorem 5. Assume that $\left(X,\|\cdot\|_{X}\right)$ does not have finite cotype. By the Maurey-Pisier theorem [MP76], for every $\theta>0$ and $m \in \mathbb{N}$ there exists a linear operator $S_{m}: \ell_{\infty}^{m} \rightarrow X$ such that

$$
\forall y \in \ell_{\infty}^{m}, \quad\|y\|_{\infty} \leqslant\left\|\mathrm{S}_{m} y\right\|_{X} \leqslant(1+\theta)\|y\|_{\infty} .
$$


Let $n \in \mathbb{N}$ with $d \in\{1, \ldots, n\}$. Define the function $f:\{-1,1\}^{n} \rightarrow L_{\infty}\left(\{-1,1\}^{n} ; \mathbb{R}\right)$ by

$$
[f(\varepsilon)](\delta) \stackrel{\text { def }}{=} T_{d}\left(\frac{\varepsilon_{1} \delta_{1}+\cdots \varepsilon_{n} \delta_{n}}{n}\right)
$$

where $T_{d}(x)$ is the $d$-th Chebyshev polynomial of the first kind and consider the composition $F:\{-1,1\}^{n} \rightarrow X$ given by $F=\mathrm{S}_{2^{n}} \circ f$, where $L_{\infty}\left(\{-1,1\}^{n} ; \mathbb{R}\right)$ is naturally identified with $\ell_{\infty}^{2^{n}}$. Since $T_{d}(x)$ is a polynomial of degree $d$, the functions $f$ and $F$ are also of degree at most $d$ and

$$
\|F(\varepsilon)\|_{X} \stackrel{(85)}{\leqslant}(1+\theta)\|f(\varepsilon)\|_{L_{\infty}\left(\{-1,1\}^{n} ; \mathbb{R}\right)}=(1+\theta) \max _{\delta \in\{-1,1\}^{n}} T_{d}\left(\frac{\varepsilon_{1} \delta_{1}+\cdots \varepsilon_{n} \delta_{n}}{n}\right)=1+\theta,
$$

for every $\varepsilon \in\{-1,1\}^{n}$, because $\left\|T_{d}\right\|_{\mathcal{e}([-1,1])}=T_{d}(1)=1$. Therefore, we also have

$$
\|F\|_{L_{p}\left(\{-1,1\}^{n} ; X\right)}=\left(\frac{1}{2^{n}} \sum_{\varepsilon \in\{-1,1\}^{n}}\|F(\varepsilon)\|_{X}^{p}\right)^{1 / p} \leqslant 1+\theta .
$$

Furthermore, for every $\varepsilon, \delta \in\{-1,1\}^{n}$,

$$
\left[\partial_{i} f(\varepsilon)\right](\delta)=\frac{1}{2}\left(T_{d}\left(\frac{\varepsilon_{1} \delta_{1}+\cdots+\varepsilon_{n} \delta_{n}}{n}\right)-T_{d}\left(\frac{\varepsilon_{1} \delta_{1}+\cdots+\varepsilon_{n} \delta_{n}}{n}-\frac{2 \varepsilon_{i} \delta_{i}}{n}\right)\right),
$$

which implies the identity

$$
[\Delta f(\varepsilon)](\delta)=\frac{1}{2}\left(n T_{d}\left(\frac{\varepsilon_{1} \delta_{1}+\cdots+\varepsilon_{n} \delta_{n}}{n}\right)-\sum_{i=1}^{n} T_{d}\left(\frac{\varepsilon_{1} \delta_{1}+\cdots+\varepsilon_{n} \delta_{n}}{n}-\frac{2 \varepsilon_{i} \delta_{i}}{n}\right)\right) .
$$

Therefore, taking $\varepsilon=\delta$, we deduce that

$$
\|\Delta f(\varepsilon)\|_{L_{\infty}\left(\{-1,1\}^{n} ; \mathbb{R}\right)} \geqslant \frac{n}{2}\left(T_{d}(1)-T_{d}\left(1-\frac{2}{n}\right)\right)=\frac{n}{2}\left(1-T_{d}\left(1-\frac{2}{n}\right)\right),
$$

from which we derive the estimate

$$
\|\Delta F\|_{L_{p}\left(\{-1,1\}^{n} ; X\right)} \stackrel{(85)}{\geqslant}\|\Delta f\|_{L_{p}\left(\{-1,1\}^{n} ; L_{\infty}\left(\{-1,1\}^{n} ; \mathbb{R}\right)\right)} \stackrel{(91)}{\geqslant} \frac{n}{2}\left(1-T_{d}\left(1-\frac{2}{n}\right)\right) .
$$

Applying (14) for the function $F:\{-1,1\}^{n} \rightarrow X$ and using (88) and (92), we finally get

$$
\begin{aligned}
\frac{n}{2}\left(1-T_{d}\left(1-\frac{2}{n}\right)\right) & \stackrel{(92)}{\leqslant}\|\Delta F\|_{L_{p}\left(\{-1,1\}^{n} ; X\right)} \\
& \stackrel{(14)}{\leqslant}(1-\eta) d^{2}\|F\|_{L_{p}\left(\{-1,1\}^{n} ; X\right)} \stackrel{(88)}{\leqslant}(1-\eta)(1+\theta) d^{2} .
\end{aligned}
$$

Letting $n \rightarrow \infty$, we get

$$
d^{2}=T_{d}^{\prime}(1) \leqslant(1-\eta)(1+\theta) d^{2} .
$$

Finally, letting $\theta \rightarrow 0^{+}$, (94) becomes $d^{2} \leqslant(1-\eta) d^{2}$ which is a contradiction.

Remark 24. Considering the function $f:\{-1,1\}^{n} \rightarrow \mathbb{C}$ given by $f(\varepsilon)=T_{d}\left(\frac{\varepsilon_{1}+\cdots+\varepsilon_{n}}{n}\right)$ for $n \rightarrow \infty$ shows that Theorem 4 is also sharp when $X=\mathbb{C}$ and $p=\infty$.

\section{Estimates for $K$-COnvex Banach spaces}

The main vector valued Fourier analytic tool which we will exploit in this section is the following fact which lies at the heart of the proof of Pisier's $K$-convexity theorem [Pis82].

Theorem 25 (Pisier). A Banach space $\left(X,\|\cdot\|_{X}\right)$ is $K$-convex if and only if for every $p \in(1, \infty)$ there exist $\theta=\theta(p, X) \in\left(0, \frac{\pi}{2}\right]$ and $M=M(p, X) \in[1, \infty)$ such that for every $n \in \mathbb{N}$

$$
|\arg z| \leqslant \theta \quad \Longrightarrow \quad\left\|e^{-z \Delta}\right\|_{L_{p}\left(\{-1,1\}^{n} ; X\right) \rightarrow L_{p}\left(\{-1,1\}^{n} ; X\right)} \leqslant M,
$$

where $\|T\|_{L_{p}\left(\{-1,1\}^{n} ; X\right) \rightarrow L_{p}\left(\{-1,1\}^{n} ; X\right)}$ is the operator norm of $T$ from $L_{p}\left(\{-1,1\}^{n} ; X\right)$ to itself. 
The fact that a Banach space satisfying (95) for some $\theta \in\left(0, \frac{\pi}{2}\right]$ and $M \in[1, \infty)$ is $K$-convex is simple. Indeed, let $a=\frac{\pi}{\tan \theta}$ so that every point $z$ in the interval with endpoints $a+i \pi, a-i \pi$ has $|\arg z| \leqslant \theta$. Then, for $k \in\{0,1, \ldots, n\}$,

$$
\frac{1}{2 \pi} \int_{-\pi}^{\pi} e^{i k t} e^{-(a+i t) \Delta} \mathrm{d} t=\frac{1}{2 \pi} \int_{-\pi}^{\pi} \sum_{j=0}^{n} e^{i k t-(a+i t) j} \operatorname{Rad}_{j} \mathrm{~d} t=e^{-k a} \operatorname{Rad}_{k},
$$

which implies that

$$
\begin{gathered}
\left\|\operatorname{Rad}_{k}\right\|_{L_{p}\left(\{-1,1\}^{n} ; X\right) \rightarrow L_{p}\left(\{-1,1\}^{n} ; X\right)}=e^{k a}\left\|\frac{1}{2 \pi} \int_{-\pi}^{\pi} e^{i k t} e^{-(a+i t) \Delta} \mathrm{d} t\right\|_{L_{p}\left(\{-1,1\}^{n} ; X\right) \rightarrow L_{p}\left(\{-1,1\}^{n} ; X\right)} \\
\leqslant \frac{e^{k a}}{2 \pi} \int_{-\pi}^{\pi}\left\|e^{-(a+i t) \Delta}\right\|_{L_{p}\left(\{-1,1\}^{n} ; X\right) \rightarrow L_{p}\left(\{-1,1\}^{n} ; X\right)} \mathrm{d} t \stackrel{(95)}{\leqslant} M e^{k a} .
\end{gathered}
$$

In particular, $\sup _{n \in \mathbb{N}}\|\operatorname{Rad}\|_{L_{p}\left(\{-1,1\}^{n} ; X\right) \rightarrow L_{p}\left(\{-1,1\}^{n} ; X\right)} \leqslant M e^{a}<\infty$, i.e. $\left(X,\|\cdot\|_{X}\right)$ is $K$-convex.

Here and throughout, we will denote by $\mathbb{D}=\{z \in \mathbb{C}:|z|<1\}$ the open unit disc. For $r \in[1, \infty)$ consider the lens domain

$$
\Omega(r) \stackrel{\text { def }}{=}\left\{z \in \mathbb{C}: \max \left\{\left|z-i \sqrt{r^{2}-1}\right|,\left|z+i \sqrt{r^{2}-1}\right|\right\}<r\right\} \subseteq \mathbb{D}
$$

and notice that $\partial \Omega(r)$ is a Jordan curve with an interior angle $\theta(r)=2 \arcsin (1 / r)$ at $z= \pm 1$. We will need the following simple consequence of Theorem 25 .

Corollary 26. Let $\left(X,\|\cdot\|_{X}\right)$ be a $K$-convex Banach space and $p \in(1, \infty)$. Then there exist some $r=r(p, X) \in[1, \infty)$ and $K=K(p, X) \in[1, \infty)$ such that

$$
\sup _{w \in \Omega(r)} \sup _{n \in \mathbb{N}}\left\|w^{\Delta}\right\|_{L_{p}\left(\{-1,1\}^{n} ; X\right) \rightarrow L_{p}\left(\{-1,1\}^{n} ; X\right)} \leqslant K .
$$

Proof. By Theorem 25 and (36), for every $w \in\left\{e^{-z}:|\arg z|<\theta\right\}$, we have

$$
\left\|w^{\Delta}\right\|_{L_{p}\left(\{-1,1\}^{n} ; X\right) \rightarrow L_{p}\left(\{-1,1\}^{n} ; X\right)}=\left\|(-w)^{\Delta}\right\|_{L_{p}\left(\{-1,1\}^{n} ; X\right) \rightarrow L_{p}\left(\{-1,1\}^{n} ; X\right)} \leqslant M .
$$

Moreover, by (97), we get

$$
\begin{aligned}
& \left\|w^{\Delta}\right\|_{L_{p}\left(\{-1,1\}^{n} ; X\right) \rightarrow L_{p}\left(\{-1,1\}^{n} ; X\right)} \\
& \quad \leqslant \sum_{k=0}^{n}|w|^{k}\left\|\operatorname{Rad}_{k}\right\|_{L_{p}\left(\{-1,1\}^{n} ; X\right) \rightarrow L_{p}\left(\{-1,1\}^{n} ; X\right)} \stackrel{(97)}{\leqslant} M \sum_{k=0}^{n}\left(|w| e^{a}\right)^{k},
\end{aligned}
$$

which implies that for $|w|<e^{-2 a}$, we also have the estimate

$$
\left\|w^{\Delta}\right\|_{L_{p}\left(\{-1,1\}^{n} ; X\right) \rightarrow L_{p}\left(\{-1,1\}^{n} ; X\right)} \leqslant \frac{M}{1-e^{-a}} .
$$

Combining the domains for which (100) and (102) hold, one can easily deduce that there exists $r \in[1, \infty)$ such that

$$
\Omega(r) \subseteq\left\{e^{-z}:|\arg z|<\theta\right\} \cup\left\{-e^{-z}:|\arg z|<\theta\right\} \cup\left\{w \in \mathbb{D}:|w|<e^{-2 a}\right\}
$$

and (99) follows with $K=\frac{M}{1-e^{-a}}$.

We record for ease of future reference the following calculation of conformal mappings.

Lemma 27. For $\alpha \in(0, \infty)$, let $\phi_{\alpha}: \mathbb{C} \backslash(-\infty, 0] \rightarrow \mathbb{C}$ be a holomorphic branch of $z \mapsto z^{\alpha}$. Also, consider the Möbius transformations $\psi_{1}(z)=\frac{1-z}{1+z}$ and $\psi_{2}(z)=\frac{z+1}{z-1}$. Then, for every $r \in[1, \infty)$, the map $\psi_{1} \circ \phi_{\pi / \theta(r)} \circ \psi_{1}$ is a conformal equivalence between $\Omega(r)$ and the unit disc $\mathbb{D}$. Furthermore, the map $\psi_{2} \circ \phi_{\pi /(2 \pi-\theta(r))} \circ \psi_{2}$ is a conformal equivalence between the complement $\overline{\Omega(r)}^{c}$ of $\overline{\Omega(r)}$ and the complement $\overline{\mathbb{D}}^{c}$ of the closed unit disc. 
Proof. Since $\psi_{1}(-1)=0, \psi_{1}(1)=\infty$ and Möbius transformations preserve angles, we have

$$
\psi_{1}(\Omega(r))=\left\{z \in \mathbb{C}:|\arg z|<\frac{\theta(r)}{2}\right\} .
$$

Therefore, composing with $\phi_{\pi / \theta(r)}$, we get

$$
\phi_{\pi / \theta(r)} \circ \psi_{1}(\Omega(r))=\{z \in \mathbb{C}: \operatorname{Re} z>0\},
$$

which immediately implies that $\psi_{1} \circ \phi_{\pi / \theta(r)} \circ \psi_{1}(\Omega(r))=\mathbb{D}$. Since all the functions involved are conformal equivalences at their domains of definition the proof is complete. The proof of the claim for the complement $\overline{\Omega(r)}^{c}$ of $\overline{\Omega(r)}$ is identical.

We can now proceed with the proof of Theorem 6 . The crucial proposition is the following.

Proposition 28. Fix a $K$-convex Banach space $\left(X,\|\cdot\|_{X}\right), p \in(1, \infty)$ and $K \in(0, \infty)$. Let $\Omega \subseteq \mathbb{D}$ be a simply connected domain bounded by a Jordan curve $\gamma$ such that $(-1,1) \subseteq \Omega$ and suppose that

$$
\sup _{w \in \Omega} \sup _{n \in \mathbb{N}}\left\|w^{\Delta}\right\|_{L_{p}\left(\{-1,1\}^{n} ; X\right) \rightarrow L_{p}\left(\{-1,1\}^{n} ; X\right)} \leqslant K .
$$

Also, let $\phi_{\Omega}: \Omega \rightarrow \mathbb{D}$ be a conformal mapping of $\Omega$ onto $\mathbb{D}$ with $\phi_{\Omega}(0)=0$ which extends continuously as a homeomorphism between $\Omega \cup \gamma$ and $\overline{\mathbb{D}}$. Then, for every $n, d \in \mathbb{N}$ with $d \in\{0,1, \ldots, n-1\}$ and every function $f:\{-1,1\}^{n} \rightarrow X$ in the $d$-th tail space, we have

$$
\forall t \geqslant 0, \quad\left\|e^{-t \Delta} f\right\|_{L_{p}\left(\{-1,1\}^{n} ; X\right)} \leqslant K\left|\phi_{\Omega}\left(e^{-t}\right)\right|^{d}\|f\|_{L_{p}\left(\{-1,1\}^{n} ; X\right)} .
$$

Proof. Fix $n \in \mathbb{N}, d \in\{0,1, \ldots, n-1\}$ and let $f:\{-1,1\}^{n} \rightarrow X$ be a function in the $d$-th tail space. For $t \geqslant 0$, consider the linear functional $\zeta_{t}:\left(\operatorname{span}\left\{w^{d}, w^{d+1}, \ldots, w^{n}\right\},\|\cdot\|_{\mathcal{C}(\bar{\Omega})}\right) \rightarrow \mathbb{C}$ given by

$$
\zeta_{t}\left(\sum_{k=d}^{n} a_{k} w^{k}\right) \stackrel{\text { def }}{=} \sum_{k=d}^{n} a_{k} e^{-t k},
$$

or $\zeta_{t}(p)=p\left(e^{-t}\right)$ if $p(w)$ is a polynomial of degree $n$ which is a multiple of $w^{d}$. If $p$ is such a polynomial, consider the function $h: \Omega \rightarrow \mathbb{C}$ given by

$$
\forall w \in \Omega, \quad h(w) \stackrel{\text { def }}{=} \frac{p(w)}{\phi_{\Omega}(w)^{d}} .
$$

Notice that $\phi_{\Omega}$ does not vanish on $\Omega \backslash\{0\}$ and furthermore it has a single root at 0 , therefore the multiplicity of the root 0 in the numerator is at least the multiplicity in the denominator. Thus $h$ is a holomorphic function on $\Omega$. Furthermore, for $w \in \gamma$, we have $\left|\phi_{\Omega}(w)\right|=1$ since $\phi_{\Omega}$ is a homeomorphism between $\gamma$ and $\partial \mathbb{D}$ (such a conformal map $\phi_{\Omega}$ always exists by Caratheodory's theorem, see [Kra06, Theorem 5.1.1]). Therefore, by the maximum principle,

$$
\sup _{w \in \Omega}|h(w)|=\max _{w \in \gamma}|h(w)|=\max _{w \in \gamma} \frac{|p(w)|}{\left|\phi_{\Omega}(w)\right|^{d}}=\|p\|_{\mathcal{C}(\gamma)}=\|p\|_{\mathcal{C}(\bar{\Omega})},
$$

which implies that for $w \in \bar{\Omega}$, we have

$$
|p(w)| \leqslant\left|\phi_{\Omega}(w)\right|^{d}\|p\|_{\mathcal{C}(\bar{\Omega})} .
$$

Applying (111) for $w=e^{-t} \in \Omega$, we deduce that the linear functional $\zeta_{t}$ satisfies

$$
\forall p \in \operatorname{span}\left\{w^{d}, w^{d+1}, \ldots, w^{n}\right\}, \quad\left|\zeta_{t}(p)\right| \leqslant\left|\phi_{\Omega}\left(e^{-t}\right)\right|^{d}\|p\|_{\mathcal{e}(\bar{\Omega})} .
$$

Therefore, by the Hahn-Banach theorem and the Riesz representation theorem, there exists a complex measure $\tau_{t}$ on $\bar{\Omega}$ such that $\left\|\tau_{t}\right\|_{\mathcal{M}(\bar{\Omega})} \leqslant\left|\phi_{\Omega}\left(e^{-t}\right)\right|^{d}$ and

$$
p \in \operatorname{span}\left\{w^{d}, w^{d+1}, \ldots, w^{n}\right\} \quad \Longrightarrow \quad \int_{\bar{\Omega}} p(w) \mathrm{d} \tau_{t}(w)=p\left(e^{-t}\right) .
$$


Since $\widehat{f}(A)=0$ when $|A|<d$, we get that

$$
\begin{aligned}
\left\|e^{-t \Delta} f\right\|_{L_{p}\left(\{-1,1\}^{n} ; X\right)} & \stackrel{(113)}{=}\left\|\int_{\bar{\Omega}} w^{\Delta} f \mathrm{~d} \tau_{t}(w)\right\|_{L_{p}\left(\{-1,1\}^{n} ; X\right)} \leqslant \int_{\bar{\Omega}}\left\|w^{\Delta} f\right\|_{L_{p}\left(\{-1,1\}^{n} ; X\right)} \mathrm{d}\left|\tau_{t}\right|(w) \\
& \stackrel{(106)}{\leqslant} K \int_{\bar{\Omega}}\|f\|_{L_{p}\left(\{-1,1\}^{n} ; X\right)} \mathrm{d}\left|\tau_{t}\right|(w)=K\left\|\tau_{t}\right\|_{\mathcal{M}(\bar{\Omega})}\|f\|_{L_{p}\left(\{-1,1\}^{n} ; X\right)} \\
& \stackrel{(112))}{\leqslant} K\left|\phi_{\Omega}\left(e^{-t}\right)\right|^{d}\|f\|_{L_{p}\left(\{-1,1\}^{n} ; X\right)},
\end{aligned}
$$

which concludes the proof.

Proof of Theorem 6. Fix $n \in \mathbb{N}, d \in\{0,1, \ldots, n-1\}, p \in(1, \infty)$ and let $f:\{-1,1\}^{n} \rightarrow X$ be a function in the $d$-th tail space. Using the notation introduced after Theorem 25 , for every $t \geqslant 2 a$,

$$
\left\|e^{-t \Delta} f\right\|_{L_{p}\left(\{-1,1\}^{n} ; X\right)} \leqslant \sum_{k=d}^{n} e^{-t k}\left\|\operatorname{Rad}_{k} f\right\|_{L_{p}\left(\{-1,1\}^{n} ; X\right)} \stackrel{(97)}{\leqslant} K e^{-\frac{1}{2} t d}\|f\|_{L_{p}\left(\{-1,1\}^{n} ; X\right)},
$$

where $K=\frac{M}{1-e^{-a}}$. We will now treat the range $t \leqslant 2 a$. By Corollary 26 , there exists $r=r(p, X) \in$ $[1, \infty)$ such that

$$
\sup _{w \in \Omega(r)} \sup _{n \in \mathbb{N}}\left\|w^{\Delta}\right\|_{L_{p}\left(\{-1,1\}^{n} ; X\right) \rightarrow L_{p}\left(\{-1,1\}^{n} ; X\right)} \leqslant K,
$$

where $\Omega(r)$ is the domain (98). Therefore, by Proposition 28, we have

$$
\forall t \geqslant 0, \quad\left\|e^{-t \Delta} f\right\|_{L_{p}\left(\{-1,1\}^{n} ; X\right)} \leqslant K\left|\phi_{\Omega(r)}\left(e^{-t}\right)\right|^{d}\|f\|_{L_{p}\left(\{-1,1\}^{n} ; X\right)} .
$$

To conclude the proof, we will show that there exists $c=c(p, X)$ such that

$$
\forall 0 \leqslant t \leqslant 2 a, \quad\left|\phi_{\Omega(r)}\left(e^{-t}\right)\right| \leqslant e^{-c t^{\pi / \theta(r)}},
$$

where $\theta(r)=2 \arcsin (1 / r) \in(0, \pi]$ which would then imply the conclusion of Theorem 6 with $A(p, X)=\frac{\pi}{\theta(r(p, X))} \in[1, \infty)$. By Lemma 27 , if $\theta=\theta(r)$, we have

$$
\phi_{\Omega(r)}\left(e^{-t}\right)=\frac{\left(1+e^{-t}\right)^{\pi / \theta}-\left(1-e^{-t}\right)^{\pi / \theta}}{\left(1+e^{-t}\right)^{\pi / \theta}+\left(1-e^{-t}\right)^{\pi / \theta}} .
$$

Therefore,

$$
\log \left|\phi_{\Omega(r)}\left(e^{-t}\right)\right|=\log \left(1-\frac{2\left(1-e^{-t}\right)^{\pi / \theta}}{\left(1+e^{-t}\right)^{\pi / \theta}+\left(1-e^{-t}\right)^{\pi / \theta}}\right) \leqslant-\frac{2\left(1-e^{-t}\right)^{\pi / \theta}}{\left(1+e^{-t}\right)^{\pi / \theta}+\left(1-e^{-t}\right)^{\pi / \theta}}
$$

and for $t \leqslant 2 a$,

$$
-\frac{2\left(1-e^{-t}\right)^{\pi / \theta}}{\left(1+e^{-t}\right)^{\pi / \theta}+\left(1-e^{-t}\right)^{\pi / \theta}} \leqslant-2^{1-\frac{\pi}{\theta}}\left(1-e^{-t}\right)^{\pi / \theta} \leqslant \frac{-2^{1-\frac{\pi}{\theta}}\left(1-e^{-2 a}\right)^{\pi / \theta}}{(2 a)^{\pi / \theta}} t^{\pi / \theta},
$$

which implies (118) with $c=\frac{2^{1-\frac{\pi}{\theta}}\left(1-e^{-2 a}\right)^{\pi / \theta}}{(2 a)^{\pi / \theta}}$.

We now proceed to prove the dual of Theorem 6, namely the improved lower bound on the decay of the heat semigroup, Theorem 7. Even though Theorems 6 and 7 are not formally dual to one another, there is a continuous analogy between the techniques used in their proofs. We will need the following elementary result from complex analysis.

Lemma 29. Let $\Omega \subseteq \mathbb{C}$ be a bounded simply connected domain and consider a conformal mapping $\phi_{\Omega^{c}}: \bar{\Omega}^{c} \rightarrow \overline{\mathbb{D}}^{c}$ of the complement of $\bar{\Omega}$ onto the complement of the closed unit disc with $\phi_{\Omega^{c}}(\infty)=$ $\infty$. Then,

$$
\lim _{z \rightarrow \infty} \frac{\phi_{\Omega^{c}}(z)}{z}=\beta \in \mathbb{C} \backslash\{0\} .
$$


Proof. Without loss of generality assume that $0 \in \Omega$ and let $V \stackrel{\text { def }}{=}\left\{z \in \mathbb{C}: \frac{1}{z} \in \bar{\Omega}^{c}\right\}$. Then $V \cup\{0\}$ is a domain and the function $F: V \rightarrow \mathbb{C}$ given by

$$
\forall z \in V, \quad F(z) \stackrel{\text { def }}{=} \frac{1}{\phi_{\Omega^{c}}(1 / z)}
$$

is holomorphic, injective and satisfies $|F(z)| \leqslant 1$ for every $z \in V$. Therefore, by Riemann's theorem on removable singularities, $F$ can be holomorphically extended at 0 . Furthermore, $F(0)=0$, since otherwise $\lim _{z \rightarrow \infty} \phi_{\Omega^{c}}(z) \in \mathbb{C}$. Since $F$ is injective, we also have $F^{\prime}(0) \neq 0$. Thus,

$$
\lim _{z \rightarrow \infty} \frac{\phi_{\Omega^{c}}(z)}{z}=\lim _{w \rightarrow 0} w \phi_{\Omega^{c}}(1 / w)=\lim _{w \rightarrow 0} \frac{w}{F(w)}=\frac{1}{F^{\prime}(0)} \in \mathbb{C} \backslash\{0\},
$$

which concludes the proof.

Proposition 30. Fix a $K$-convex Banach space $\left(X,\|\cdot\|_{X}\right), p \in(1, \infty)$ and $K \in(0, \infty)$. Let $\Omega \subseteq \mathbb{D}$ be a simply connected domain bounded by a Jordan curve $\gamma$ and suppose that

$$
\sup _{w \in \Omega} \sup _{n \in \mathbb{N}}\left\|w^{\Delta}\right\|_{L_{p}\left(\{-1,1\}^{n} ; X\right) \rightarrow L_{p}\left(\{-1,1\}^{n} ; X\right)} \leqslant K .
$$

Also, let $\phi_{\Omega^{c}}: \bar{\Omega}^{c} \rightarrow \overline{\mathbb{D}}^{c}$ be a conformal mapping of the complement of $\bar{\Omega}$ onto the complement of the closed unit disc which extends continuously as a homeomorphism between $\Omega^{c}$ and $\mathbb{D}^{c}$. Then, for every $n, d \in \mathbb{N}$ with $d \in\{1, \ldots, n\}$ and every function $f:\{-1,1\}^{n} \rightarrow X$ of degree at most $d$, we have

$$
\forall t \geqslant 0, \quad\left\|e^{-t \Delta} f\right\|_{L_{p}\left(\{-1,1\}^{n} ; X\right)} \geqslant \frac{1}{K\left|\phi_{\Omega^{c}}\left(e^{t}\right)\right|^{d}}\|f\|_{L_{p}\left(\{-1,1\}^{n} ; X\right)} .
$$

Proof. Fix $n \in \mathbb{N}, d \in\{1, \ldots, n\}$ and let $f:\{-1,1\}^{n} \rightarrow X$ be a function of degree at most $d$. For $t \geqslant 0$, consider the linear functional $\varphi_{t}:\left(\operatorname{span}\left\{1, w, \ldots, w^{d}\right\},\|\cdot\|_{\mathcal{C}(\bar{\Omega})}\right) \rightarrow \mathbb{C}$ given by

$$
\varphi_{t}\left(\sum_{k=0}^{d} a_{k} w^{k}\right) \stackrel{\text { def }}{=} \sum_{k=0}^{d} a_{k} e^{t k}
$$

or $\varphi_{t}(p)=p\left(e^{t}\right)$ when $p$ is a polynomial with $\operatorname{deg}(p) \leqslant d$. If $p$ is such a polynomial, consider the function $h: \bar{\Omega}^{c} \rightarrow \mathbb{C}$ given by

$$
\forall z \in \bar{\Omega}^{c}, \quad h(z) \stackrel{\text { def }}{=} \frac{p(z)}{\phi_{\Omega^{c}}(z)^{d}} .
$$

Notice that, since the conformal map $\phi_{\Omega^{c}}$ extends as a homeomorphism between $\Omega^{c}$ and $\mathbb{D}^{c}$, it also satisfies $\phi_{\Omega^{c}}(\infty)=\infty$. Thus, (122) implies that $\lim _{z \rightarrow \infty} h(z) \in \mathbb{C}$ and therefore $h$ is a holomorphic function on $\bar{\Omega}^{c} \cup\{\infty\}$. Furthermore, for $z \in \gamma$, we have $\left|\phi_{\Omega^{c}}(z)\right|=1$ since $\phi_{\Omega^{c}}$ is a homeomorphism between $\gamma$ and $\partial \mathbb{D}$. Therefore, by the maximum principle,

$$
\sup _{z \in \Omega^{c}}|h(z)|=\max _{z \in \gamma}|h(z)|=\max _{z \in \gamma} \frac{|p(z)|}{\left|\phi_{\Omega^{c}}(z)\right|^{d}}=\|p\|_{\mathcal{C}(\gamma)}=\|p\|_{\mathcal{C}(\bar{\Omega})},
$$

which implies that for $z \in \Omega^{c}$, we have

$$
|p(z)| \leqslant\left|\phi_{\Omega^{c}}(z)\right|^{d}\|p\|_{\mathcal{e}(\bar{\Omega})} .
$$

Applying (130) for $z=e^{t} \in \Omega^{c}$, we deduce that the linear functional $\varphi_{t}$ satisfies

$$
\forall p \in \operatorname{span}\left\{1, w, \ldots, w^{d}\right\}, \quad\left|\varphi_{t}(p)\right| \leqslant\left|\phi_{\Omega^{c}}\left(e^{t}\right)\right|^{d}\|p\|_{\mathcal{C}(\bar{\Omega})} .
$$

Therefore, by the Hahn-Banach theorem and the Riesz representation theorem, there exists a complex measure $\rho_{t}$ on $\bar{\Omega}$ such that $\left\|\rho_{t}\right\|_{\mathcal{M}(\bar{\Omega})} \leqslant\left|\phi_{\Omega^{c}}\left(e^{t}\right)\right|^{d}$ and

$$
p \in \operatorname{span}\left\{1, w, \ldots, w^{d}\right\} \quad \Longrightarrow \quad \int_{\bar{\Omega}} p(w) \mathrm{d} \rho_{t}(w)=p\left(e^{t}\right) .
$$


Since $\widehat{f}(A)=0$ when $|A|>d$, we get that

$$
\begin{aligned}
\left\|e^{t \Delta} f\right\|_{L_{p}\left(\{-1,1\}^{n} ; X\right)} & \stackrel{(132)}{=}\left\|\int_{\bar{\Omega}} w^{\Delta} f \mathrm{~d} \rho_{t}(w)\right\|_{L_{p}\left(\{-1,1\}^{n} ; X\right)} \leqslant \int_{\bar{\Omega}}\left\|w^{\Delta} f\right\|_{L_{p}\left(\{-1,1\}^{n} ; X\right)} \mathrm{d}\left|\rho_{t}\right|(w) \\
& \stackrel{(125)}{\leqslant} K \int_{\bar{\Omega}}\|f\|_{L_{p}\left(\{-1,1\}^{n} ; X\right)} \mathrm{d}\left|\rho_{t}\right|(w)=K\left\|\rho_{t}\right\|_{\mathcal{M}(\bar{\Omega})}\|f\|_{L_{p}\left(\{-1,1\}^{n} ; X\right)} \\
& \stackrel{(131)}{\leqslant} K\left|\phi_{\Omega^{c}}\left(e^{t}\right)\right|^{d}\|f\|_{L_{p}\left(\{-1,1\}^{n} ; X\right)},
\end{aligned}
$$

which is equivalent to (126).

Proof of Theorem 7. Fix $n \in \mathbb{N}, d \in\{1, \ldots, n\}, p \in(1, \infty)$ and let $f:\{-1,1\}^{n} \rightarrow X$ be a function of degree at most $d$. By Theorem 1 , for every $t \geqslant 0$,

$$
\left\|e^{-t \Delta} f\right\|_{L_{p}\left(\{-1,1\}^{n} ; X\right)} \geqslant \frac{1}{T_{d}\left(e^{t}\right)}\|f\|_{L_{p}\left(\{-1,1\}^{n} ; X\right)}
$$

and, by (44), for $t \geqslant 1$,

$$
T_{d}\left(e^{t}\right) \stackrel{(44)}{\leqslant}\left(2 e^{t}\right)^{d} \leqslant e^{2 t d} .
$$

We will now treat the range $t \leqslant 1$. By Corollary 26 , there exists $r=r(p, X) \in[1, \infty)$ such that

$$
\sup _{w \in \Omega(r)} \sup _{n \in \mathbb{N}}\left\|w^{\Delta}\right\|_{L_{p}\left(\{-1,1\}^{n} ; X\right) \rightarrow L_{p}\left(\{-1,1\}^{n} ; X\right)} \leqslant K,
$$

where $\Omega(r)$ is the domain (98). Therefore, by Proposition 30, we have

$$
\forall t \geqslant 0, \quad\left\|e^{-t \Delta} f\right\|_{L_{p}\left(\{-1,1\}^{n} ; X\right)} \geqslant \frac{1}{K\left|\phi_{\Omega(r)^{c}}\left(e^{t}\right)\right|^{d}}\|f\|_{L_{p}\left(\{-1,1\}^{n} ; X\right)} .
$$

To conclude the proof, we will show that there exists $C=C(p, X)$ such that

$$
\forall 0 \leqslant t \leqslant 1, \quad\left|\phi_{\Omega(r)^{c}}\left(e^{t}\right)\right| \leqslant e^{C t^{\pi /(2 \pi-\theta(r))}},
$$

where $\theta(r)=2 \arcsin (1 / r) \in(0, \pi]$ which would then imply the conclusion of Theorem 7 with $\eta(p, X)=\frac{\pi}{2 \pi-\theta(r(p, X))} \in\left(\frac{1}{2}, 1\right]$. By Lemma 27, if $\theta=\theta(r)$, we have

$$
\phi_{\Omega(r)^{c}}\left(e^{t}\right)=\frac{\left(e^{t}+1\right)^{\frac{\pi}{2 \pi-\theta}}+\left(e^{t}-1\right)^{\frac{\pi}{2 \pi-\theta}}}{\left(e^{t}+1\right)^{\frac{\pi}{2 \pi-\theta}}-\left(e^{t}-1\right)^{\frac{\pi}{2 \pi-\theta}}} .
$$

Therefore,

$$
\log \left|\phi_{\Omega(r)^{c}}\left(e^{t}\right)\right|=\log \left(1+\frac{2\left(e^{t}-1\right)^{\frac{\pi}{2 \pi-\theta}}}{\left(e^{t}+1\right)^{\frac{\pi}{2 \pi-\theta}}-\left(e^{t}-1\right)^{\frac{\pi}{2 \pi-\theta}}}\right) \leqslant \frac{2\left(e^{t}-1\right)^{\frac{\pi}{2 \pi-\theta}}}{\left(e^{t}+1\right)^{\frac{\pi}{2 \pi-\theta}}-\left(e^{t}-1\right)^{\frac{\pi}{2 \pi-\theta}}}
$$

and for $t \leqslant 1$,

$$
\frac{2\left(e^{t}-1\right)^{\frac{\pi}{2 \pi-\theta}}}{\left(e^{t}+1\right)^{\frac{\pi}{2 \pi-\theta}}-\left(e^{t}-1\right)^{\frac{\pi}{2 \pi-\theta}}} \leqslant \frac{2\left(e^{t}-1\right)^{\frac{\pi}{2 \pi-\theta}}}{(e+1)^{\frac{\pi}{2 \pi-\theta}}-(e-1)^{\frac{\pi}{2 \pi-\theta}}} \leqslant \frac{2(e-1)^{\frac{\pi}{2 \pi-\theta}}}{(e+1)^{\frac{\pi}{2 \pi-\theta}}-(e-1)^{\frac{\pi}{2 \pi-\theta}}} t^{\frac{\pi}{2 \pi-\theta}}
$$

which implies $(138)$ with $C=\frac{2(e-1)^{\frac{\pi}{2 \pi-\theta}}}{(e+1)^{\frac{\pi}{2 \pi-\theta}}-(e-1)^{\frac{\pi}{2 \pi-\theta}}}$.

An argument identical to the one used in the proof of Corollary 2 implies the following improved moment comparison of low degree functions with values in a $K$-convex space $\left(X,\|\cdot\|_{X}\right)$. 
Corollary 31. Let $\left(X,\|\cdot\|_{X}\right)$ be a $K$-convex Banach space. For every $p>q>1$, there exist $C=C(p, X) \in(0, \infty)$ and $\eta=\eta(p, X) \in\left(\frac{1}{2}, 1\right]$ such that for every $n, d \in \mathbb{N}$ with $d \in\{1, \ldots, n\}$ and every function $f:\{-1,1\}^{n} \rightarrow X$ of degree at most $d$, we have

$$
\|f\|_{L_{p}\left(\{-1,1\}^{n} ; X\right)} \leqslant C e^{C d \max \left\{\log \left(\frac{p-1}{q-1}\right), \log \left(\frac{p-1}{q-1}\right)^{\eta}\right\}}\|f\|_{L_{q}\left(\{-1,1\}^{n} ; X\right)} .
$$

We now proceed to prove Theorem 8, the improved Bernstein-Markov inequality for the Laplacian. For this, we will use the following classical approximation theoretic result of Szegö [Sze25].

Theorem 32 (Szegö). Let $\Omega \subseteq \mathbb{C}$ be a domain bounded by a Jordan curve $\gamma$ and fix $w \in \gamma$. Denote by $\theta(\Omega, w) \in[0,2 \pi]$ the exterior angle of $\gamma$ at $w$. Then, there exists a constant $K(\Omega, w) \in(0, \infty)$ such that for every $d \in \mathbb{N}$ and every polynomial $p$ of degree at most $d$, we have

$$
\left|p^{\prime}(w)\right| \leqslant K(\Omega, w) d^{\theta(\Omega, w) / \pi}\|p\|_{\mathcal{C}(\bar{\Omega})} .
$$

A simple proof of Szegö's theorem specifically for lens domains of the form (98) was given in [EI18].

Proof of Theorem 8. Fix $n \in \mathbb{N}, d \in\{1, \ldots, n\}, p \in(1, \infty)$ and let $f:\{-1,1\}^{n} \rightarrow X$ be a function of degree at most $d$. Also let $\theta=\theta(p, X) \in\left(0, \frac{\pi}{2}\right]$ and $M=M(p, X) \in[1, \infty)$ be given by Theorem 25. Consider the domain $V=\left\{e^{-z}:|\arg z|<\theta\right\} \subseteq \mathbb{D}$. Then, by Theorem 25, we have

$$
\forall w \in V, \quad\left\|w^{\Delta}\right\|_{L_{p}\left(\{-1,1\}^{n} ; X\right) \rightarrow L_{p}\left(\{-1,1\}^{n} ; X\right)} \leqslant M .
$$

As in the proof of Theorem 4 , consider the linear functional $\psi:\left(\operatorname{span}\left\{1, w, \ldots, w^{d}\right\},\|\cdot\|_{\mathfrak{e}(\bar{V})}\right) \rightarrow \mathbb{C}$ given by

$$
\psi\left(\sum_{k=0}^{d} a_{k} w^{k}\right) \stackrel{\text { def }}{=} \sum_{k=0}^{d} k a_{k},
$$

or $\psi(p)=p^{\prime}(1)$ when $p$ is a polynomial with $\operatorname{deg}(p) \leqslant d$. Notice that $V$ is a domain bounded by a Jordan curve $\gamma$ with $1 \in \gamma$ which forms an exterior angle $2 \pi-2 \theta$ at 1 . Then, Szegö's inequality (143) implies that there exists some $K=K(p, X) \in(0, \infty)$ such that

$$
\forall p \in \operatorname{span}\left\{1, w, \ldots, w^{d}\right\}, \quad|\psi(p)| \leqslant K d^{2-\frac{2 \theta}{\pi}}\|p\|_{\mathcal{e}(\bar{V})} .
$$

Therefore, by the Hahn-Banach theorem and the Riesz representation theorem, there exists a complex measure $v$ on $\bar{V}$ such that $\|v\|_{\mathcal{M}(\bar{V})} \leqslant K d^{2-\frac{2 \theta}{\pi}}$ such that for every polynomial $p$, we have

$$
\operatorname{deg}(p) \leqslant d \quad \Longrightarrow \quad \int_{\bar{V}} p(w) \mathrm{d} v(w)=p^{\prime}(1)
$$

Since $\widehat{f}(A)=0$ when $|A|>d$, we get that

$$
\begin{aligned}
\|\Delta f\|_{L_{p}\left(\{-1,1\}^{n} ; X\right)} \stackrel{(147)}{=}\left\|\int_{\bar{V}} w^{\Delta} f \mathrm{~d} v(w)\right\|_{L_{p}\left(\{-1,1\}^{n} ; X\right)} \leqslant \int_{\bar{V}}\left\|w^{\Delta} f\right\|_{L_{p}\left(\{-1,1\}^{n} ; X\right)} \mathrm{d}|v|(w) \\
\stackrel{(95)}{\leqslant} M \int_{\bar{V}}\|f\|_{L_{p}\left(\{-1,1\}^{n} ; X\right)} \mathrm{d}|v|(w) \stackrel{(146)}{\leqslant} K M d^{2-\frac{2 \theta}{\pi}}\|f\|_{L_{p}\left(\{-1,1\}^{n} ; X\right)},
\end{aligned}
$$

which concludes the proof of the theorem with $\alpha(p, X)=2-\frac{2 \theta(p, X)}{\pi} \in[1,2)$.

Remark 33. It is straightfoward to see that if the Bernstein-Markov inequality (12) holds true with linear dependence on the degree for a given Banach space, then the asymptotically optimal lower bound for the action of the heat semigroup conjectured after Theorem 7 follows. Indeed, assume that for a Banach space $\left(X,\|\cdot\|_{X}\right)$ and $p \in[1, \infty)$ there exists $C \in(0, \infty)$ such that for every $n, d \in \mathbb{N}$ with $d \in\{1, \ldots, n\}$ and every function $f:\{-1,1\}^{n} \rightarrow X$ of degree at most $d$, we have

$$
\|\Delta f\|_{L_{p}\left(\{-1,1\}^{n} ; X\right)} \leqslant C d\|f\|_{L_{p}\left(\{-1,1\}^{n} ; X\right)}
$$


Then, for every $t \geqslant 0$, we get

$$
\begin{aligned}
\left\|e^{t \Delta} f\right\|_{L_{p}\left(\{-1,1\}^{n} ; X\right)} \leqslant \sum_{k=0}^{\infty} \frac{\left\|(t \Delta)^{k} f\right\|_{L_{p}\left(\{-1,1\}^{n} ; X\right)}}{k !} \stackrel{(149)}{\leqslant} \sum_{k=0}^{\infty} \frac{(C t d)^{k}}{k !}\|f\|_{L_{p}\left(\{-1,1\}^{n} ; X\right)} \\
=e^{C t d}\|f\|_{L_{p}\left(\{-1,1\}^{n} ; X\right)}
\end{aligned}
$$

which, applied to $e^{-t \Delta} f$, is equivalent to the conjectured optimal version of Theorem 7 .

The Bernstein-Markov inequality (20) for the vector valued gradient is an immediate consequence of Theorem 8 combined with the following dual to Pisier's inequality (11) for low degree functions.

Proposition 34. Let $\left(X,\|\cdot\|_{X}\right)$ be a $K$-convex Banach space. For every $p \in(1, \infty)$ there exists $B(p, X) \in(0, \infty)$ such that for every $n, d \in \mathbb{N}$ with $d \in\{1, \ldots, n\}$, every function $g:\{-1,1\}^{n} \rightarrow X$ of degree at most d satisfies

$$
\left(\frac{1}{2^{n}} \sum_{\delta \in\{-1,1\}^{n}}\left\|\sum_{i=1}^{n} \delta_{i} \partial_{i} g\right\|_{L_{p}\left(\{-1,1\}^{n} ; X\right)}^{p}\right)^{1 / p} \leqslant B(p, X)(\log d+1)\|\Delta g\|_{L_{p}\left(\{-1,1\}^{n} ; X\right)} .
$$

Proof. Following the notation (71) of the proof of Theorem 3, for $t \geqslant 0$, consider the function $g_{t}:\{-1,1\}^{n} \times\{-1,1\}^{n} \rightarrow X$ given by

$$
\begin{aligned}
g_{t}(\varepsilon, \delta) & \stackrel{\text { def }}{=} \sum_{A \subseteq\{1, \ldots, n\}} \widehat{g}(A) \prod_{i \in A}\left(e^{-t} \varepsilon_{i}+\left(1-e^{-t}\right) \delta_{i}\right) \\
& =e^{-t \Delta} g(\varepsilon)+\left(e^{t}-1\right) \sum_{i=1}^{n} \varepsilon_{i} \delta_{i} \partial_{i} e^{-t \Delta} g(\varepsilon)+\Phi_{t}(\varepsilon, \delta),
\end{aligned}
$$

where $\sum_{\delta \in\{-1,1\}^{n}} \delta_{i} \Phi_{t}(\varepsilon, \delta)=0$ for every $\varepsilon \in\{-1,1\}^{n}$ and $i \in\{1, \ldots, n\}$. Therefore, for every $t>0$, we have

$$
\begin{aligned}
\left(\frac{1}{2^{n}} \sum_{\delta \in\{-1,1\}^{n}}\left\|\sum_{i=1}^{n} \delta_{i} \partial_{i} e^{-t \Delta} g\right\|_{L_{p}\left(\{-1,1\}^{n} ; X\right)}^{p}\right)^{1 / p} \stackrel{(152)}{=} \frac{1}{e^{t}-1}\left\|\operatorname{Rad}_{\delta} g_{t}\right\|_{L_{p}\left(\{-1,1\}^{n} \times\{-1,1\}^{n} ; X\right)} \\
\leqslant \frac{K}{e^{t}-1}\left\|g_{t}\right\|_{L_{p}\left(\{-1,1\}^{n} \times\{-1,1\}^{n} ; X\right)} \stackrel{(73)}{\leqslant} \frac{K}{e^{t}-1}\|g\|_{L_{p}\left(\{-1,1\}^{n} ; X\right)},
\end{aligned}
$$

where $\operatorname{Rad}_{\delta} g_{t}$ is the Rademacher projection of $g_{t}$ with respect to the variable $\delta \in\{-1,1\}^{n}, K=$ $K(p, X)=\sup _{n \in \mathbb{N}}\|\operatorname{Rad}\|_{L_{p}\left(\{-1,1\}^{n} ; X\right) \rightarrow L_{p}\left(\{-1,1\}^{n} ; X\right)}<\infty$ and the proof of the last inequality is identical to the proof of (73) via (72). Integrating the above inequality, we deduce that for $s>0$,

$$
\begin{aligned}
\left(\frac{1}{2^{n}} \sum_{\delta \in\{-1,1\}^{n}} \|\right. & \left.\sum_{i=1}^{n} \delta_{i} \partial_{i} \Delta^{-1} e^{-s \Delta} g \|_{L_{p}\left(\{-1,1\}^{n} ; X\right)}^{p}\right)^{1 / p} \\
& =\left(\frac{1}{2^{n}} \sum_{\delta \in\{-1,1\}^{n}}\left\|\int_{s}^{\infty} \sum_{i=1}^{n} \delta_{i} \partial_{i} e^{-t \Delta} g \mathrm{~d} t\right\|_{L_{p}\left(\{-1,1\}^{n} ; X\right)}^{p}\right)^{1 / p} \\
& \stackrel{(153)}{\leqslant} K\left(\int_{s}^{\infty} \frac{1}{e^{t}-1} \mathrm{~d} t\right)\|g\|_{L_{p}\left(\{-1,1\}^{n} ; X\right)}=K \log \left(\frac{e^{s}}{e^{s}-1}\right)\|g\|_{L_{p}\left(\{-1,1\}^{n} ; X\right)} .
\end{aligned}
$$

By Theorem 1 and the elementary inequality $T_{d}\left(e^{s}\right) \leqslant e^{d^{2} s}$, where $s \geqslant 0$, we conclude that for every $\delta \in\{-1,1\}^{n}$,

$$
\left\|\sum_{i=1}^{n} \delta_{i} \partial_{i} \Delta^{-1} e^{-s \Delta} g\right\|_{L_{p}\left(\{-1,1\}^{n} ; X\right)} \geqslant e^{-d^{2} s}\left\|\sum_{i=1}^{n} \delta_{i} \partial_{i} \Delta^{-1} g\right\|_{L_{p}\left(\{-1,1\}^{n} ; X\right)} .
$$


Therefore, combining (154) and (155),

$$
\begin{array}{r}
\left(\frac{1}{2^{n}} \sum_{\delta \in\{-1,1\}^{n}}\left\|\sum_{i=1}^{n} \delta_{i} \partial_{i} \Delta^{-1} g\right\|_{L_{p}\left(\{-1,1\}^{n} ; X\right)}^{p}\right)^{1 / p} \leqslant K \min _{s \geqslant 0} e^{d^{2} s} \log \left(\frac{e^{s}}{e^{s}-1}\right)\|g\|_{L_{p}\left(\{-1,1\}^{n} ; X\right)} \\
\leqslant 3 K(\log d+1)\|g\|_{L_{p}\left(\{-1,1\}^{n} ; X\right)}
\end{array}
$$

which is equivalent to the desired inequality (151) with $B(p, X)=3 K(p, X)$.

Remark 35. It has been shown in [NS02] that the validity of $(151)$ with the factor $C(\log d+1)$ replaced by a constant $C(p, X)$ depending only on $p$ and the Banach space $\left(X,\|\cdot\|_{X}\right)$, where $p \in(1, \infty)$, implies that $X$ is $K$-convex. Nevertheless, (151) is the best known bound to date for general $K$-convex spaces, even when $d=n$. Under additional assumptions (e.g. when $X$ is a $\mathrm{UMD}^{+}$space or when $X$ is a $K$-convex Banach lattice), inequality (151) is known to hold true with a constant $C(p, X)$ independent of the dimension $n$ for functions of arbitrary degree $d$, see [HN13].

Proof of Theorem 9. Fix $n \in \mathbb{N}, d \in\{1, \ldots, n\}$ and let $f:\{-1,1\}^{n} \rightarrow X$ be a function of degree at most $d$. Then, we have

$$
\begin{gathered}
\left(\frac{1}{2^{n}} \sum_{\delta \in\{-1,1\}^{n}}\left\|\sum_{i=1}^{n} \delta_{i} \partial_{i} f\right\|{ }_{L_{p}\left(\{-1,1\}^{n} ; X\right)}\right)^{1 / p} \stackrel{(151)}{\leqslant} B(p, X)(\log d+1)\|\Delta f\|_{L_{p}\left(\{-1,1\}^{n} ; X\right)} \\
\stackrel{(19)}{\leqslant} B(p, X) C(p, X) d^{\alpha(p, X)}(\log d+1)\|f\|_{L_{p}\left(\{-1,1\}^{n} ; X\right)},
\end{gathered}
$$

which completes the proof.

Finally, we will prove the reverse Bernstein-Markov inequality of Theorem 10. Recall that for $\gamma \in(0, \infty)$ and a function $f:\{-1,1\}^{n} \rightarrow X$, we denote by

$$
\Delta^{\gamma} f \stackrel{\text { def }}{=} \sum_{A \subseteq\{1, \ldots, n\}}|A|^{\gamma} \widehat{f}(A) w_{A}
$$

the action of a fractional power of the hypercube Laplacian $\Delta$ on $f$. We will prove the following statement for $\Delta^{1 / 2}$ of which Theorem 10 is an immediate consequence.

Theorem 36. Let $\left(X,\|\cdot\|_{X}\right)$ be a $K$-convex Banach space. For every $p \in(1, \infty)$ there exists $c(p, X) \in(0, \infty)$ such that for every $n, d, m \in \mathbb{N}$ with $d+m \leqslant n$ and every function $f:\{-1,1\}^{n} \rightarrow X$ of degree at most $d+m$ which is also in the $d$-th tail space, we have

$$
\left\|\Delta^{1 / 2} f\right\|_{L_{p}\left(\{-1,1\}^{n} ; X\right)} \geqslant c(p, X) \sqrt{\frac{d}{m}}\|f\|_{L_{p}\left(\{-1,1\}^{n} ; X\right)} .
$$

We start by proving the following lemma.

Lemma 37. Let $\left(X,\|\cdot\|_{X}\right)$ be a $K$-convex Banach space. For every $p \in(1, \infty)$ there exists $K=K(p, X) \in(0, \infty)$ such that for every $n \in \mathbb{N}$ and every function $f:\{-1,1\}^{n} \rightarrow X$, we have

$$
\forall t \geqslant 0, \quad\left\|\Delta^{1 / 2} e^{-t \Delta} f\right\|_{L_{p}\left(\{-1,1\}^{n} ; X\right)} \leqslant \frac{K}{\sqrt{e^{2 t}-1}}\|f\|_{L_{p}\left(\{-1,1\}^{n} ; X\right)} .
$$

Proof. By (97), there exist $M=M(p, X) \in(0, \infty)$ and $a=a(p, X) \in(0, \infty)$ such that for every $n \in \mathbb{N}, k \in\{1, \ldots, n\}$ and every function $f:\{-1,1\}^{n} \rightarrow X$, we have

$$
\left\|\operatorname{Rad}_{k} f\right\|_{L_{p}\left(\{-1,1\}^{n} ; X\right)} \leqslant M e^{k a}\|f\|_{L_{p}\left(\{-1,1\}^{n} ; X\right)} .
$$


Therefore, for $t \geqslant 2 a$,

$$
\begin{aligned}
& \left\|\Delta^{1 / 2} e^{-t \Delta} f\right\|_{L_{p}\left(\{-1,1\}^{n} ; X\right)} \leqslant \sum_{k=1}^{n} \sqrt{k} e^{-t k}\left\|\operatorname{Rad}_{k} f\right\|_{L_{p}\left(\{-1,1\}^{n} ; X\right)} \\
& \quad \stackrel{(161)}{\leqslant} M \sum_{k=1}^{n} \sqrt{k} e^{-(t-a) k}\|f\|_{L_{p}\left(\{-1,1\}^{n} ; X\right)} \leqslant\left(M e^{a} \sum_{k=1}^{\infty} \sqrt{k} e^{-a k}\right) e^{-t}\|f\|_{L_{p}\left(\{-1,1\}^{n} ; X\right)},
\end{aligned}
$$

which implies that for $t \geqslant 2 a$,

$$
\left\|\Delta^{1 / 2} e^{-t \Delta} f\right\|_{L_{p}\left(\{-1,1\}^{n} ; X\right)} \leqslant \frac{K}{\sqrt{e^{2 t}-1}}\|f\|_{L_{p}\left(\{-1,1\}^{n} ; X\right)},
$$

where $K=M e^{a} \sum_{k=1}^{\infty} \sqrt{k} e^{-a k}$. To prove $(160)$ for $t \in(0,2 a)$, recall that by Pisier's $K$-convexity theorem, there exists $\theta=\theta(p, X) \in\left(0, \frac{\pi}{2}\right]$ such that

$$
|\arg z| \leqslant \theta \quad \Longrightarrow \quad\left\|e^{-z \Delta}\right\|_{L_{p}\left(\{-1,1\}^{n} ; X\right) \rightarrow L_{p}\left(\{-1,1\}^{n} ; X\right)} \leqslant M
$$

Let $r=t \sin \theta$ and notice that the closed disc $\bar{D}(t, r)$ of radius $r$ centered at $t$ is contained in $\{z \in \mathbb{C}:|\arg z| \leqslant \theta\}$. By the Cauchy integral formula for the derivative,

$$
-\Delta e^{-t \Delta}=\frac{1}{2 \pi i} \int_{\partial D(t, r)} \frac{e^{-\zeta \Delta}}{(\zeta-t)^{2}} \mathrm{~d} \zeta
$$

hence

$$
\left\|\Delta e^{-t \Delta} f\right\|_{L_{p}\left(\{-1,1\}^{n} ; X\right)} \stackrel{(165)}{\leqslant} \frac{1}{r} \sup _{\zeta \in \partial D(t, r)}\left\|e^{-\zeta \Delta} f\right\|_{L_{p}\left(\{-1,1\}^{n} ; X\right)} \stackrel{(164)}{\leqslant} \frac{M}{t \sin \theta}\|f\|_{L_{p}\left(\{-1,1\}^{n} ; X\right)} .
$$

Furthermore, by an inequality of Naor and Schechtman [BELP08, Lemma 5.6], for every $\beta \in(0,1)$ and function $g:\{-1,1\}^{n} \rightarrow X$, we have

$$
\left\|\Delta^{\beta} g\right\|_{L_{p}\left(\{-1,1\}^{n} ; X\right)} \leqslant 4\|\Delta g\|_{L_{p}\left(\{-1,1\}^{n} ; X\right)}^{\beta}\|g\|_{L_{p}\left(\{-1,1\}^{n} ; X\right)}^{1-\beta} .
$$

Therefore, combining (167) for $g=e^{-t \Delta} f$ and $\beta=\frac{1}{2}$ with (166) and the contractivity of the heat semigroup, we deduce that

$$
\left\|\Delta^{1 / 2} e^{-t \Delta} f\right\|_{L_{p}\left(\{-1,1\}^{n} ; X\right)} \leqslant \frac{4 \sqrt{M}}{\sqrt{t} \sqrt{\sin \theta}}\|f\|_{L_{p}\left(\{-1,1\}^{n} ; X\right)},
$$

which completes the proof of $(160)$, since for every $a \in(0, \infty)$, there exists $c_{a} \in(0, \infty)$ such that $\sqrt{e^{2 t}-1} \leqslant c_{a} \sqrt{t}$ for $t \in(0,2 a)$.

In the proof of Theorem 36, we will use a reverse Bernstein inequality for incomplete polynomials, proven recently by Erdélyi [Erd20].

Theorem 38 (Erdélyi). Fix $d, m \in \mathbb{N}$ and let $P(x)$ be a polynomial of the form

$$
P(x)=a_{d} x^{d}+a_{d+1} x^{d+1}+\cdots+a_{d+m} x^{d+m} .
$$

Then,

$$
|P(1)| \leqslant 6 \sqrt{\frac{m}{d}}\left\|\sqrt{1-x^{2}} P^{\prime}(x)\right\|_{\mathcal{e}([0,1])} .
$$

Furthermore, the estimate is sharp up to the value of the universal constant. 
Proof of Theorem 36. Fix $n, d, m \in \mathbb{N}$ with $d+m \leqslant n$ and a function $f:\{-1,1\}^{n} \rightarrow X$ of degree at most $d+m$ which is also in the $d$-th tail space. Writing $x=e^{-t} \in[0,1],(160)$ can be rewritten as

$$
\forall x \in[0,1], \quad\left\|\sqrt{1-x^{2}} \Delta x^{\Delta-1} f\right\|_{L_{p}\left(\{-1,1\}^{n} ; X\right)} \leqslant K\left\|\Delta^{1 / 2} f\right\|_{L_{p}\left(\{-1,1\}^{n} ; X\right)} .
$$

Consider the linear functional $\xi:\left(\operatorname{span}\left\{\sqrt{1-x^{2}} x^{d-1}, \ldots, \sqrt{1-x^{2}} x^{d+m-1}\right\},\|\cdot\|_{\mathcal{e}([0,1])}\right)$ given by

$$
\xi\left(\sqrt{1-x^{2}} \sum_{k=d}^{d+m} k a_{k} x^{k-1}\right) \stackrel{\text { def }}{=} \sum_{k=d}^{d+m} a_{k} .
$$

Then, Erdélyi's inequality (170) can be rewritten as

$$
\forall p \in \operatorname{span}\left\{\sqrt{1-x^{2}} x^{d-1}, \ldots, \sqrt{1-x^{2}} x^{d+m-1}\right\}, \quad|\xi(p)| \leqslant 6 \sqrt{\frac{m}{d}}\|p\|_{\mathcal{e}([0,1])} .
$$

Therefore, by the Hahn-Banach theorem and the Riesz representation theorem, there exists a complex measure $\sigma$ on $[0,1]$ such that $\|\sigma\|_{\mathcal{M}([0,1])} \leqslant 6 \sqrt{\frac{m}{d}}$ and

$$
\forall p \in \operatorname{span}\left\{\sqrt{1-x^{2}} x^{d-1}, \ldots, \sqrt{1-x^{2}} x^{d+m-1}\right\}, \quad \int_{0}^{1} p(x) \mathrm{d} \sigma(x)=\xi(p) .
$$

Since $\widehat{f}(A)=0$ when $|A| \notin\{d, \ldots, d+m\}$, we deduce that

$$
\begin{aligned}
& \|f\|_{L_{p}\left(\{-1,1\}^{n} ; X\right)} \stackrel{(174)}{=}\left\|\int_{0}^{1} \sqrt{1-x^{2}} \Delta x^{\Delta-1} f \mathrm{~d} \sigma(x)\right\|_{L_{p}\left(\{-1,1\}^{n} ; X\right)} \\
& \quad \leqslant \int_{0}^{1}\left\|\sqrt{1-x^{2}} \Delta x^{\Delta-1} f\right\|_{L_{p}\left(\{-1,1\}^{n} ; X\right)} \mathrm{d}|\sigma|(x) \stackrel{(171)}{\leqslant} \int_{0}^{1} K\left\|\Delta^{1 / 2} f\right\|_{L_{p}\left(\{-1,1\}^{n} ; X\right)} \mathrm{d}|\sigma|(x) \\
& \quad=K\|\sigma\|_{\mathcal{M}([0,1])}\left\|\Delta^{1 / 2} f\right\|_{L_{p}\left(\{-1,1\}^{n} ; X\right)} \stackrel{(173)}{\leqslant} 6 K \sqrt{\frac{m}{d}}\left\|\Delta^{1 / 2} f\right\|_{L_{p}\left(\{-1,1\}^{n} ; X\right)},
\end{aligned}
$$

which is equivalent to (159).

Proof of Theorem 10. Fix $n, d, m \in \mathbb{N}$ with $d+m \leqslant n$ and a function $f:\{-1,1\}^{n} \rightarrow X$ of degree at most $d+m$ which is also in the $d$-th tail space. Then, Theorem 36 implies that

$$
\begin{aligned}
\|\Delta f\|_{L_{p}\left(\{-1,1\}^{n} ; X\right)} & =\left\|\Delta^{1 / 2} \Delta^{1 / 2} f\right\|_{L_{p}\left(\{-1,1\}^{n} ; X\right)} \\
& \stackrel{(159)}{\geqslant} c(p, X) \sqrt{\frac{d}{m}}\left\|\Delta^{1 / 2} f\right\|_{L_{p}\left(\{-1,1\}^{n} ; X\right)} \stackrel{(159)}{\geqslant} c(p, X)^{2} \frac{d}{m}\|f\|_{L_{p}\left(\{-1,1\}^{n} ; X\right)},
\end{aligned}
$$

which is the desired inequality.

\section{Estimates for scalar VALUed funCtions}

We noticed in Corollary 26 that Pisier's $K$-convexity theorem easily implies that for every $K$ convex Banach space $\left(X,\|\cdot\|_{X}\right)$ and $p \in(1, \infty)$ there exist $r=r(p, X) \in[1, \infty)$ and $K=K(p, X) \in$ $[1, \infty)$ such that

$$
\sup _{w \in \Omega(r)} \sup _{n \in \mathbb{N}}\left\|w^{\Delta}\right\|_{L_{p}\left(\{-1,1\}^{n} ; X\right) \rightarrow L_{p}\left(\{-1,1\}^{n} ; X\right)} \leqslant K,
$$

where the domain $\Omega(r)$ is given by (98). The scalar valued version of this result was first studied in classical work of Weissler [Wei79] who found the exact domain $\Omega_{p} \subseteq \mathbb{D}$ for which the operator $w^{\Delta}: L_{p}\left(\{-1,1\}^{n} ; \mathbb{C}\right) \rightarrow L_{p}\left(\{-1,1\}^{n} ; \mathbb{C}\right)$ is uniformly bounded (equivalently, a contraction) for $p \in(1, \infty) \backslash\left(\frac{3}{2}, 2\right) \cup(2,3)$ and $w \in \Omega_{p}$. Finally, the domain $\Omega_{p}$ for $p$ in the remaining range $\left(\frac{3}{2}, 2\right) \cup(2,3)$ was recently identified by the second named author and Nazarov in [IN19]. 
Theorem 39 (Weissler, Ivanisvili-Nazarov). For $p \in(1, \infty)$, let $r_{p}=\frac{p}{2 \sqrt{p-1}}$. Then, for every $p \in(1, \infty)$ and $w \in \mathbb{C}$, we have

$$
w \in \overline{\Omega\left(r_{p}\right)} \Longleftrightarrow \sup _{n \in \mathbb{N}}\left\|w^{\Delta}\right\|_{L_{p}\left(\{-1,1\}^{n} ; \mathbb{C}\right) \rightarrow L_{p}\left(\{-1,1\}^{n} ; \mathbb{C}\right)}=1 .
$$

Furthermore, both conditions are equivalent to

$$
\sup _{n \in \mathbb{N}}\left\|w^{\Delta}\right\|_{L_{p}\left(\{-1,1\}^{n} ; \mathbb{C}\right) \rightarrow L_{p}\left(\{-1,1\}^{n} ; \mathbb{C}\right)}<\infty .
$$

The decay properties of Theorem 11 are straightforward consequences of Theorem 39 and the results of Section 3.

Proof of Theorem 11. Inequality (23) for functions of low degree is a straightforward consequence of Theorem 39 combined with Proposition 30 and (139). Similarly, inequality (24) for functions in the tail space follows from Theorem 39 combined with Proposition 28 and (119).

Proof of Corollary 12. The deduction of Corollary 12 from Theorem 11 is identical to the deduction of Corollary 2 from Theorem 1; it follows by concatenating (23) with Bonami's hypercontractive inequality (46) and choosing $t=\frac{1}{2} \log \left(\frac{p-1}{q-1}\right)$.

We now proceed with the proof of Theorem 13, the improved $L_{1}-L_{2}$ moment comparison for low degree functions. We use the following important result of Beckner [Bec75] and Weissler [Wei79].

Theorem 40 (Beckner, Weissler). Let $p \in(2, \infty)$ and $p^{*} \in(1,2)$ its conjugate exponent, that is $p^{*}=\frac{p}{p-1}$. Then, a complex number $w \in \mathbb{D}$ satisfies

$$
\left.\max \left\{\left|w-\frac{p-2}{2(p-1)}\right|,\left|w+\frac{p-2}{2(p-1)}\right|\right\} \leqslant \frac{p}{2(p-1)}\right\}
$$

if and only if

$$
\sup _{n \in \mathbb{N}}\left\|w^{\Delta}\right\|_{L_{p}\left(\{-1,1\}^{n} ; \mathbb{C}\right) \rightarrow L_{p^{*}}\left(\{-1,1\}^{n} ; \mathbb{C}\right)}=1 .
$$

Furthermore, both conditions are equivalent to

$$
\sup _{n \in \mathbb{N}}\left\|w^{\Delta}\right\|_{L_{p}\left(\{-1,1\}^{n} ; \mathbb{C}\right) \rightarrow L_{p^{*}}\left(\{-1,1\}^{n} ; \mathbb{C}\right)}<\infty .
$$

In [Bec75], Beckner proved that $w= \pm i \sqrt{p^{*}-1}$ satisfies (181) and then Weissler [Wei79] modified his argument to obtain (181) for $w$ in the full domain(180).

Proof of Theorem 13. Fix $n \in \mathbb{N}$ and $d \in\{1, \ldots, n\}$. For $p \in(2, \infty)$, denote by $V_{p} \subseteq \mathbb{D}$ the set of all $w \in \mathbb{D}$ that satisfy (180). Then, a straightforward computation shows that

$$
i \sqrt{p-1} V_{p}=\overline{\Omega\left(\frac{p}{2 \sqrt{p-1}}\right)}
$$

where $\Omega(r)$ is defined by (98). Therefore, by Lemma 27 , the function $\varphi_{p}: V_{p}^{c} \rightarrow \overline{\mathbb{D}}^{c}$

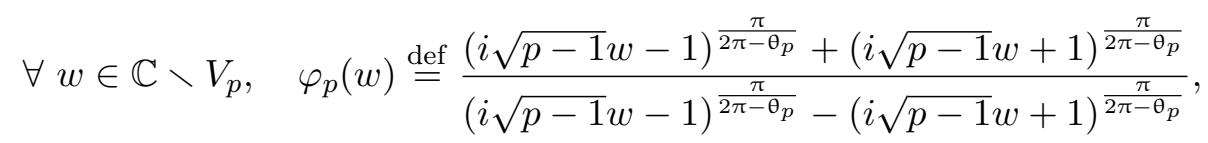

where $\theta_{p}=2 \arcsin \left(\frac{2 \sqrt{p-1}}{p}\right)$, is a conformal equivalence between the complement of $V_{p}$ and the complement of the closed unit disc $\overline{\mathbb{D}}$. A duality argument identical to that of Proposition 30 combined with (181) now implies that for every function $f:\{-1,1\}^{n} \rightarrow \mathbb{C}$ of degree at most $d$,

$$
\forall t \geqslant 0, \quad\left\|e^{-t \Delta} f\right\|_{L_{p^{*}}\left(\{-1,1\}^{n} ; \mathbb{C}\right)} \geqslant \frac{1}{\left|\varphi_{p}\left(e^{t}\right)\right|^{d}}\|f\|_{L_{p}\left(\{-1,1\}^{n} ; \mathbb{C}\right)} .
$$


In particular, for $t=0$, we have

$$
\|f\|_{L_{p}\left(\{-1,1\}^{n} ; \mathbb{C}\right)} \leqslant\left|\varphi_{p}(1)\right|^{d}\|f\|_{L_{p^{*}}\left(\{-1,1\}^{n} ; \mathbb{C}\right)} .
$$

Now, by Hölder's inequality, we get

$$
\|f\|_{L_{2}\left(\{-1,1\}^{n} ; \mathbb{C}\right)} \leqslant\|f\|_{L_{1}\left(\{-1,1\}^{n} ; \mathbb{C}\right)}^{\frac{p-2}{2(p-1)}}\|f\|_{L_{p}\left(\{-1,1\}^{n} ; \mathbb{C}\right)}^{\frac{p}{2(-1)}}
$$

and

$$
\|f\|_{L_{p^{*}}\left(\{-1,1\}^{n} ; \mathbb{C}\right)} \leqslant\|f\|_{L_{1}\left(\{-1,1\}^{n} ; \mathbb{C}\right)}^{\frac{p-2}{p-1}}\|f\|_{L_{p}\left(\{-1,1\}^{n} ; \mathbb{C}\right)}^{\frac{1}{p-1}}
$$

Combining the two, we deduce that

$$
\begin{aligned}
&\|f\|_{L_{2}\left(\{-1,1\}^{n} ; \mathbb{C}\right)} \leqslant\|f\|_{L_{1}\left(\{-1,1\}^{n} ; \mathbb{C}\right)}\left(\frac{\|f\|_{L_{p}\left(\{-1,1\}^{n} ; \mathbb{C}\right)}}{\|f\|_{L_{p^{*}}\left(\{-1,1\}^{n} ; \mathbb{C}\right)}}\right) \stackrel{p}{\frac{p}{2(p-2)}} \\
& \stackrel{(186)}{\leqslant}\left|\varphi_{p}(1)\right|^{\frac{p d}{2(p-2)}}\|f\|_{L_{1}\left(\{-1,1\}^{n} ; \mathbb{C}\right)} .
\end{aligned}
$$

Consequently,

where

$$
\|f\|_{L_{2}\left(\{-1,1\}^{n} ; \mathbb{C}\right)} \leqslant C^{d}\|f\|_{L_{1}\left(\{-1,1\}^{n} ; \mathbb{C}\right)}
$$

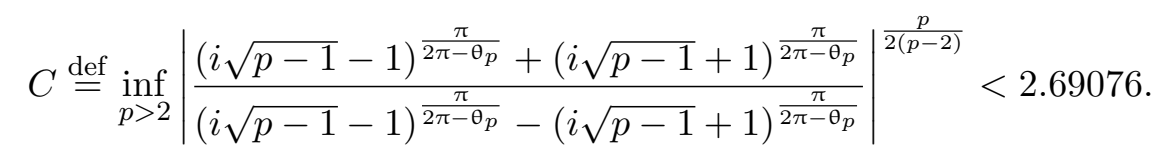

The last inequality can be checked numerically.

We note in passing that the bound $\|f\|_{L_{2}\left(\{-1,1\}^{n} ; \mathbb{C}\right)} \leqslant e^{d / 2}\|f\|_{L_{1}\left(\{-1,1\}^{n} ; \mathbb{C}\right)}$, which improves upon Theorem 13, was obtained in the recent work [IT19] for $d$-homogeneous functions $f:\{-1,1\}^{n} \rightarrow \mathbb{C}$.

Remark 41. Following Beckner's pioneering work [Bec75], significant efforts were devoted in identifying the complex domains consisting of those $w \in \mathbb{D}$ for which

$$
\sup _{n \in \mathbb{N}}\left\|w^{\Delta}\right\|_{L_{p}\left(\{-1,1\}^{n} ; \mathbb{C}\right) \rightarrow L_{q}\left(\{-1,1\}^{n} ; \mathbb{C}\right)}<\infty
$$

for general $p \geqslant q>1$. In [Wei79], Weissler managed to precisely characterize these complex numbers $w$ for all $p \geqslant q>1$ apart from the cases $\frac{3}{2}<q \leqslant p<2$ and $2<q \leqslant p<3$ and posed a conjecture for $p, q$ in the remaining ranges. The case $p=q$ of his conjecture was recently settled by the second named author and Nazarov [IN19]. In contrast to this long standing problem, Epperson [Epp89] (see also [Jan97]) has characterized those $w \in \mathbb{D}$ for which

$$
\sup _{n \in \mathbb{N}}\left\|w^{L}\right\|_{L_{p}\left(\left(\mathbb{R}^{n}, \gamma_{n}\right) ; \mathbb{C}\right) \rightarrow L_{q}\left(\left(\mathbb{R}^{n}, \gamma_{n}\right) ; \mathbb{C}\right)}<\infty
$$

where $\gamma_{n}$ is the standard Gaussian measure on $\mathbb{R}^{n}$ and $L=\Delta-\langle x, \nabla\rangle$ is the generator of the Ornstein-Uhlenbeck semigroup, for every $p>q>1$. For $p$ and $q$ not belonging in the missing ranges mentioned earlier, the domains of complex hypercontractivity for the Hamming cube and the Gauss space coincide and it is natural to believe that this is also the case when $\frac{3}{2}<q<p<2$ and $2<q<p<3$.

It is evident from the proof above that the constant 2.69076 appearing in Theorem 13 is not optimal. In fact, one can run a similar argument starting with the inequality

$$
\|f\|_{L_{2}\left(\{-1,1\}^{n} ; \mathbb{C}\right)} \leqslant\|f\|_{L_{1}\left(\{-1,1\}^{n} ; \mathbb{C}\right)}\left(\frac{\|f\|_{L_{p}\left(\{-1,1\}^{n} ; \mathbb{C}\right)}}{\|f\|_{L_{q}\left(\{-1,1\}^{n} ; \mathbb{C}\right)}}\right)^{\frac{p q}{2(p-q)}},
$$

which is valid for any $p>2>q$ and any function $f:\{-1,1\}^{n} \rightarrow \mathbb{C}$. Then, to obtain an $L_{q}-L_{p}$ moment comparison as in the proof of Theorem 13, one should use the general $L_{q}-L_{p}$ 
complex hypercontractivity of [Wei79] and explicitly compute the conformal map of the domain provided by Weissler's theorem. In fact, such a computation could also provide an improvement of Corollary 12. We did not attempt to optimize any of these computations as the domains of $L_{q}-L_{p}$ hypercontractivity for $q \notin\left\{p, p^{*}\right\}$ tend to be quite complicated. We also note that for $p>2$, the least constant $C_{p}$ for which every function $f:\{-1,1\}^{n} \rightarrow \mathbb{C}$ of degree at most $d$ satisfies

$$
\|f\|_{L_{p}\left(\{-1,1\}^{n} ; \mathbb{C}\right)} \leqslant C_{p}^{d}\|f\|_{L_{2}\left(\{-1,1\}^{n} ; \mathbb{C}\right)}
$$

is known to be $C_{p}=\sqrt{p-1}$ (see [IT19]), yet the sharp constant in (27) is still unknown. Inequality (195) with $C_{p}=\sqrt{p-1}$ is usually proven via an orthogonality argument (see [O'D14, Theorem 9.21]), but a duality based proof can be given using the result of Weissler [Wei79] who showed that for $p \geqslant 2$

$$
|w| \leqslant \frac{1}{\sqrt{p-1}} \Longleftrightarrow \sup _{n \in \mathbb{N}}\left\|w^{\Delta}\right\|_{L_{p}\left(\{-1,1\}^{n} ; \mathbb{C}\right) \rightarrow L_{2}\left(\{-1,1\}^{n} ; \mathbb{C}\right)}=1 .
$$

A straightfoward adaptation of the proof of Proposition 30 then implies that for every function $f:\{-1,1\}^{n} \rightarrow \mathbb{C}$ of degree at most $d$, we have

$$
\forall t \geqslant 0, \quad\left\|e^{-t \Delta} f\right\|_{L_{2}\left(\{-1,1\}^{n} ; \mathbb{C}\right)} \geqslant\left(\frac{e^{-t}}{\sqrt{p-1}}\right)^{d}\|f\|_{L_{p}\left(\{-1,1\}^{n} ; \mathbb{C}\right)},
$$

which for $t=0$ coincides with (195) with $C_{p}=\sqrt{p-1}$.

Proof of Theorem 14. The proof is a mechanical adaptation of the proof of Theorem 8, where (144) is replaced by the characterization (178) of the complex domain where the heat flow is a contraction. The fact that the underlying constant $K=K(\Omega, w)$ in Szegö's theorem can be taken to be the absolute constant 10 if $\Omega$ is a lens domain of the form (98) and $w=1$ was shown in [EI18, Proposition 15]. This proves the Bernstein-Markov inequality (28).

To derive the Bernstein-Markov inequalities for the discrete gradient presented in Theorem 15, we will need to make use of Lust-Piquard's Riesz transform inequalities [LP98] (see also [BELP08] where the implicit dependence in $p$ was improved).

Theorem 42 (Lust-Piquard). For every $p \in[2, \infty)$, there exist $c_{p}, C_{p} \in(0, \infty)$ such that for every $n \in \mathbb{N}$, every function $f:\{-1,1\}^{n} \rightarrow \mathbb{C}$ satisfies

$$
c_{p}\left\|\Delta^{1 / 2} f\right\|_{L_{p}\left(\{-1,1\}^{n} ; \mathbb{C}\right)} \leqslant\|\nabla f\|_{L_{p}\left(\{-1,1\}^{n} ; \mathbb{C}\right)} \leqslant C_{p}\left\|\Delta^{1 / 2} f\right\|_{L_{p}\left(\{-1,1\}^{n} ; \mathbb{C}\right)} .
$$

Proof of Theorem 15 for $p \geqslant 2$. Fix $n \in \mathbb{N}, d \in\{1, \ldots, n\}$ and let $f:\{-1,1\}^{n} \rightarrow \mathbb{C}$ be a function of degree at most $d$. Then, by Lust-Piquard's inequality (198), we have

$$
\|\nabla f\|_{L_{p}\left(\{-1,1\}^{n} ; \mathbb{C}\right)} \leqslant C_{p}\left\|\Delta^{1 / 2} f\right\|_{L_{p}\left(\{-1,1\}^{n} ; \mathbb{C}\right)} .
$$

Combining (199), Naor and Schechtman's inequality (167) for $\beta=\frac{1}{2}$ and Theorem 14, we derive the Bernstein-Markov inequalities of Theorem 15 for $p \geqslant 2$.

Even though the one-sided Riesz transform inequality

$$
\forall p \in(1, \infty), \quad c_{p}\left\|\Delta^{1 / 2} f\right\|_{L_{p}\left(\{-1,1\}^{n} ; \mathbb{C}\right)} \leqslant\|\nabla f\|_{L_{p}\left(\{-1,1\}^{n} ; \mathbb{C}\right)}
$$

is true for $p \in(1,2)$ (see [LP98]), its reverse is known to be false in this range. In fact, it has been shown by Naor and Schechtman (see [BELP08, Lemma 5.5]) that, if $p \in(1,2)$, a dimension independent inequality of the form

$$
\|\nabla f\|_{L_{p}\left(\{-1,1\}^{n} ; \mathbb{C}\right)} \leqslant C\left\|\Delta^{\beta} f\right\|_{L_{p}\left(\{-1,1\}^{n} ; \mathbb{C}\right)}
$$


implies that $\beta \geqslant \frac{1}{p}$. We will now show that this is almost optimal. The following proposition is due to A. Naor, to whom we are grateful for allowing us to include it here. The proof presented here is different than Naor's original proof, which will appear elsewhere.

Proposition 43 (Naor). For every $p \in(1,2)$ and every $\varepsilon \in\left(0, \frac{1}{2}\right)$, there exists $C_{p} \in(0, \infty)$ such that for every $n \in \mathbb{N}$, every function $f:\{-1,1\}^{n} \rightarrow \mathbb{C}$ satisfies

$$
\|\nabla f\|_{L_{p}\left(\{-1,1\}^{n} ; \mathbb{C}\right)} \leqslant \frac{C_{p}}{\varepsilon}\left\|\Delta^{1 / p+\varepsilon} f\right\|_{L_{p}\left(\{-1,1\}^{n} ; \mathbb{C}\right)} .
$$

For the proof of Proposition 43 we will need the following lemma.

Lemma 44. For every $p \in(1,2]$, there exists $A_{p} \in(0, \infty)$ such that for every $n \in \mathbb{N}$, every function $f:\{-1,1\}^{n} \rightarrow \mathbb{C}$ satisfies

$$
\forall t>0, \quad\left\|\nabla e^{-t \Delta} f\right\|_{L_{p}\left(\{-1,1\}^{n} ; \mathbb{C}\right)} \leqslant \frac{A_{p}}{\left(e^{p t}-1\right)^{1 / p}} \cdot\|f\|_{L_{p}\left(\{-1,1\}^{n} ; \mathbb{C}\right)} .
$$

Proof. For $t \geqslant 0$, consider the operator $S_{t}: L_{p}\left(\{-1,1\}^{n} ; \mathbb{C}\right) \rightarrow L_{p}\left(\{-1,1\}^{n} \times\{-1,1\}^{n} ; \mathbb{C}\right)$ given by

$$
S_{t}(f)(\varepsilon, \delta) \stackrel{\text { def }}{=} \sum_{A \subseteq\{1, \ldots, n\}} \widehat{f}(A) \prod_{i \in A}\left(e^{-t} \varepsilon_{i}+\left(1-e^{-t}\right) \delta_{i}\right)-e^{-t \Delta} f(\varepsilon),
$$

where $(\varepsilon, \delta) \in\{-1,1\}^{n} \times\{-1,1\}^{n}$. Notice that, because of $(72)$, (73) and the contractivity of the heat semigroup, we have

$$
\left\|S_{t}(f)\right\|_{L_{1}\left(\{-1,1\}^{n} \times\{-1,1\}^{n} ; \mathbb{C}\right)} \leqslant 2\|f\|_{L_{1}\left(\{-1,1\}^{n} ; \mathbb{C}\right)} .
$$

Furthermore, if $f=w_{A}$ for a subset $A \subseteq\{1, \ldots, n\}$,

$$
S_{t}\left(w_{A}\right)(\varepsilon, \delta)=\sum_{B \subsetneq A} e^{-t|B|}\left(1-e^{-t}\right)^{|A \backslash B|} w_{B}(\varepsilon) w_{A \backslash B}(\delta),
$$

which by orthogonality implies that

$$
\begin{aligned}
\left\|S_{t}\left(w_{A}\right)\right\|_{L_{2}\left(\{-1,1\}^{n} \times\{-1,1\}^{n} ; \mathbb{C}\right)}^{2} & =\sum_{k=0}^{|A|-1}\left(\begin{array}{c}
|A| \\
k
\end{array}\right) e^{-2 t k}\left(1-e^{-t}\right)^{2(|A|-k)} \\
& =\left(e^{-2 t}+\left(1-e^{-t}\right)^{2}\right)^{|A|}-e^{-2 t|A|} \leqslant 1-e^{-t},
\end{aligned}
$$

where the last inequality is elementary. Therefore,

$$
\begin{gathered}
\left\|S_{t}\right\|_{L_{2}\left(\{-1,1\}^{n} ; \mathbb{C}\right) \rightarrow L_{2}\left(\{-1,1\}^{n} \times\{-1,1\}^{n} ; \mathbb{C}\right)}=\max _{A \subseteq\{1, \ldots, n\}}\left\|S_{t}\left(w_{A}\right)\right\|_{L_{2}\left(\{-1,1\}^{n} \times\{-1,1\}^{n} ; \mathbb{C}\right)} \\
\leqslant \sqrt{1-e^{-t}} .
\end{gathered}
$$

Using the Riesz-Thorin interpolation theorem, we conclude that for every $p \in[1,2]$,

$$
\left\|S_{t}\right\|_{L_{p}\left(\{-1,1\}^{n} ; \mathbb{C}\right) \rightarrow L_{p}\left(\{-1,1\}^{n} \times\{-1,1\}^{n} ; \mathbb{C}\right)} \stackrel{(205) \wedge(208)}{\leqslant} 2^{\frac{2}{p}-1}\left(1-e^{-t}\right)^{1-\frac{1}{p}} \leqslant 2\left(1-e^{-t}\right)^{1-\frac{1}{p}}
$$

and, by (71), we get

$$
\begin{aligned}
& \left(\frac{1}{2^{n}} \sum_{\delta \in\{-1,1\}^{n}}\left\|\sum_{i=1}^{n} \delta_{i} \partial_{i} e^{-t \Delta} f\right\|_{L_{p}\left(\{-1,1\}^{n} ; \mathbb{C}\right)}^{p}\right)^{1 / p} \stackrel{(71)}{=} \frac{1}{e^{t}-1}\left\|\operatorname{Rad}_{\delta} S_{t}(f)\right\|_{L_{p}\left(\{-1,1\}^{n} \times\{-1,1\}^{n} ; \mathbb{C}\right)} \\
& \leqslant \frac{K_{p}}{e^{t}-1}\left\|S_{t}(f)\right\|_{L_{p}\left(\{-1,1\}^{n} \times\{-1,1\}^{n} ; \mathbb{C}\right)} \stackrel{(209)}{\leqslant} \frac{2 K_{p}\|f\|_{L_{p}\left(\{-1,1\}^{n} ; \mathbb{C}\right)}}{e^{t\left(1-\frac{1}{p}\right)}\left(e^{t}-1\right)^{\frac{1}{p}}} \leqslant \frac{4 K_{p}\|f\|_{L_{p}\left(\{-1,1\}^{n} ; \mathbb{C}\right)}}{\left(e^{p t}-1\right)^{\frac{1}{p}}},
\end{aligned}
$$


where $K_{p}=\sup _{n \in \mathbb{N}}\|\operatorname{Rad}\|_{L_{p}\left(\{-1,1\}^{n} ; \mathbb{C}\right) \rightarrow L_{p}\left(\{-1,1\}^{n} ; \mathbb{C}\right)}$. Finally, by Khintchine's inequality,

$$
\left\|\nabla e^{-t \Delta} f\right\|_{L_{p}\left(\{-1,1\}^{n} ; \mathbb{C}\right)} \leqslant B_{p}\left(\frac{1}{2^{n}} \sum_{\delta \in\{-1,1\}^{n}}\left\|\sum_{i=1}^{n} \delta_{i} \partial_{i} e^{-t \Delta} f\right\|_{L_{p}\left(\{-1,1\}^{n} ; \mathbb{C}\right)}^{p}\right)^{1 / p}
$$

for some $B_{p} \in[1, \sqrt{2}]$, which combined with (210) completes the proof.

Proof of Proposition 43. Fix $\varepsilon \in\left(0, \frac{1}{2}\right), n \in \mathbb{N}, d \in\{1, \ldots, n\}$ and let $f:\{-1,1\}^{n} \rightarrow \mathbb{C}$. A change of variables gives the integral representation

$$
\Delta^{-1 / p-\varepsilon} f=\frac{1}{\Gamma\left(\frac{1}{p}+\varepsilon\right)} \int_{0}^{\infty} t^{\frac{1}{p}+\varepsilon-1} e^{-t \Delta} f \mathrm{~d} t
$$

Therefore, we can write

$$
\begin{aligned}
\left\|\nabla \Delta^{-1 / p-\varepsilon} f\right\|_{L_{p}\left(\{-1,1\}^{n} ; \mathbb{C}\right)} & \stackrel{(212)}{=} \frac{1}{\Gamma\left(\frac{1}{p}+\varepsilon\right)}\left\|\nabla\left(\int_{0}^{\infty} t^{\frac{1}{p}+\varepsilon-1} e^{-t \Delta} f \mathrm{~d} t\right)\right\|_{L_{p}\left(\{-1,1\}^{n} ; \mathbb{C}\right)} \\
& \leqslant \frac{1}{\Gamma\left(\frac{1}{p}+\varepsilon\right)} \int_{0}^{\infty} t^{\frac{1}{p}+\varepsilon-1}\left\|\nabla e^{-t \Delta} f\right\|_{L_{p}\left(\{-1,1\}^{n} ; \mathbb{C}\right)} \mathrm{d} t \\
& \stackrel{(203)}{\leqslant} \frac{A_{p}}{\Gamma(1 / 2)}\left(\int_{0}^{\infty} \frac{t^{\frac{1}{p}+\varepsilon-1}}{\left(e^{p t}-1\right)^{1 / p}} \mathrm{~d} t\right)\|f\|_{L_{p}\left(\{-1,1\}^{n} ; \mathbb{C}\right) .}
\end{aligned}
$$

To conclude the proof, notice that

$$
\int_{0}^{\infty} \frac{t^{\frac{1}{p}+\varepsilon-1}}{\left(e^{p t}-1\right)^{1 / p}} \mathrm{~d} t=\int_{0}^{1} \frac{t^{\frac{1}{p}+\varepsilon-1}}{\left(e^{p t}-1\right)^{1 / p}} \mathrm{~d} t+\int_{1}^{\infty} \frac{t^{\frac{1}{p}+\varepsilon-1}}{\left(e^{p t}-1\right)^{1 / p}} \mathrm{~d} t=O(1 / \varepsilon)+O(1)=O(1 / \varepsilon)
$$

and thus (213) becomes

$$
\left\|\nabla \Delta^{-1 / p-\varepsilon} f\right\|_{L_{p}\left(\{-1,1\}^{n} ; \mathbb{C}\right)} \leqslant \frac{C_{p}}{\varepsilon}\|f\|_{L_{p}\left(\{-1,1\}^{n} ; \mathbb{C}\right)},
$$

which is equivalent to (202).

Remark 45. Combining (202) with (167) and the estimate $\|\Delta f\|_{L_{p}\left(\{-1,1\}^{n} ; \mathbb{C}\right)} \leqslant n\|f\|_{L_{p}\left(\{-1,1\}^{n} ; \mathbb{C}\right)}$ which holds true for every function $f:\{-1,1\}^{n} \rightarrow \mathbb{C}$, we get

$$
\|\nabla f\|_{L_{p}\left(\{-1,1\}^{n} ; \mathbb{C}\right)} \leqslant \frac{4 C_{p} n^{\varepsilon}}{\varepsilon}\left\|\Delta^{1 / p} f\right\|_{L_{p}\left(\{-1,1\}^{n} ; \mathbb{C}\right)},
$$

which for $\varepsilon=\frac{1}{\log (n+1)}$ becomes

$$
\|\nabla f\|_{L_{p}\left(\{-1,1\}^{n} ; \mathbb{C}\right)} \leqslant 4 e C_{p} \log (n+1)\left\|\Delta^{1 / p} f\right\|_{L_{p}\left(\{-1,1\}^{n} ; \mathbb{C}\right)} .
$$

In the upcoming manuscript [EN20], the first named author and Naor use a new Littlewood-PaleyStein inequality [Ste70] on the discrete hypercube, which allows to improve the logarithmic term in $(217)$ to $(\log n)^{c_{p}}$ for some $c_{p} \in(0,1)$, where $p \in(1,2)$. We conjecture that (217) holds true with a dimension independent constant.

Proof of Theorem 15 for $p \in(1,2)$. Fix $\varepsilon \in(0,1), n \in \mathbb{N}, d \in\{1, \ldots, n\}$ and let $f:\{-1,1\}^{n} \rightarrow \mathbb{C}$ be a function of degree at most $d$. Combining (202), (167) for $\beta=\frac{1}{p}+\varepsilon$ and (28), we deduce that

$$
\|\nabla f\|_{L_{p}\left(\{-1,1\}^{n} ; \mathbb{C}\right)} \leqslant \frac{40 C_{p}}{\varepsilon} d^{\left(2-\frac{\theta_{p}}{\pi}\right)\left(\frac{1}{p}+\varepsilon\right)}\|f\|_{L_{p}\left(\{-1,1\}^{n} ; \mathbb{C}\right)} .
$$

Choosing $\varepsilon=\frac{1}{\log (d+1)}$ gives the conclusion.

The next proposition is an asymptotically sharp endpoint Bernstein-Markov inequality for the discrete gradient and $p=\infty$. 
Proposition 46. For every $n, d \in \mathbb{N}$ with $d \in\{1, \ldots, n\}$ and every function $f:\{-1,1\}^{n} \rightarrow \mathbb{C}$,

$$
\|\nabla f\|_{L_{\infty}\left(\{-1,1\}^{n} ; \mathbb{C}\right)} \leqslant 2 d\|f\|_{L_{\infty}\left(\{-1,1\}^{n} ; \mathbb{C}\right)} .
$$

Furthermore, the factor $d$ is asymptotically optimal.

We will need the following lemma, which is a special case of a more general semigroup statement from [BGL14, Proposition 8.6.1] and is the $L_{\infty}$ analogue of Lemma 44 .

Lemma 47. For every $n \in \mathbb{N}$ and every function $f:\{-1,1\}^{n} \rightarrow \mathbb{C}$, we have

$$
\forall t>0, \quad\left\|\nabla e^{-t \Delta} f\right\|_{L_{\infty}\left(\{-1,1\}^{n} ; \mathbb{C}\right)} \leqslant \frac{1}{\sqrt{e^{2 t}-1}}\|f\|_{L_{\infty}\left(\{-1,1\}^{n} ; \mathbb{C}\right)} .
$$

Proof. Fix $n \in \mathbb{N}$ and consider a function $f:\{-1,1\}^{n} \rightarrow \mathbb{C}$. Then, we have the pointwise identity

$$
\left(e^{2 t}-1\right) \sum_{i=1}^{n}\left(\partial_{i} e^{-t \Delta} f\right)^{2}=2 \int_{0}^{t} e^{2 s} \sum_{i=1}^{n}\left(\partial_{i} e^{-t \Delta} f\right)^{2} \mathrm{~d} s .
$$

Denote by $g_{i}(\varepsilon)=\varepsilon_{i} \partial_{i} e^{-(t-s) \Delta} f(\varepsilon)$ and notice that

$$
\forall \varepsilon \in\{-1,1\}^{n}, \quad \varepsilon_{i} e^{s} \partial_{i} e^{-t \Delta} f(\varepsilon)=e^{-s \Delta} g_{i}(\varepsilon) .
$$

Therefore, (221) implies that

$$
\begin{aligned}
\left(e^{2 t}-1\right) \sum_{i=1}^{n}\left(\partial_{i} e^{-t \Delta} f\right)^{2} & =2 \int_{0}^{t} \sum_{i=1}^{n}\left(e^{-s \Delta} g_{i}\right)^{2} \mathrm{~d} s \\
& \stackrel{(\dagger)}{\leqslant} 2 \int_{0}^{t} e^{-s \Delta}\left(\sum_{i=1}^{n} g_{i}^{2}\right) \mathrm{d} s=2 \int_{0}^{t} e^{-s \Delta}\left(\sum_{i=1}^{n}\left(\partial_{i} e^{-(t-s) \Delta} f\right)^{2}\right) \mathrm{d} s
\end{aligned}
$$

where inequality $(\dagger)$ follows from Jensen's inequality since $e^{-s \Delta}$ is an averaging operator. Using the definition of the Laplacian, one can check that for every function $h:\{-1,1\}^{n} \rightarrow \mathbb{C}$ the identity

$$
\forall \varepsilon \in\{-1,1\}^{n}, \quad 2 h(\varepsilon) \cdot \Delta h(\varepsilon)-\Delta h^{2}(\varepsilon)=2 \sum_{i=1}^{n}\left(\partial_{i} h(\varepsilon)\right)^{2}
$$

holds true. Combining (223) and (224), we conclude that

$$
\left(e^{2 t}-1\right) \sum_{i=1}^{n}\left(\partial_{i} e^{-t \Delta} f\right)^{2} \leqslant \int_{0}^{t} e^{-s \Delta}\left(2 e^{-(t-s) \Delta} f \cdot \Delta e^{-(t-s) \Delta} f-\Delta\left(e^{-(t-s) \Delta} f\right)^{2}\right) \mathrm{d} s .
$$

However, we also have

$$
\frac{\mathrm{d}}{\mathrm{d} s} e^{-s \Delta}\left(e^{-(t-s) \Delta} f\right)^{2}=e^{-s \Delta}\left(2 e^{-(t-s) \Delta} f \cdot \Delta e^{-(t-s) \Delta} f-\Delta\left(e^{-(t-s) \Delta} f\right)^{2}\right),
$$

which implies that (225) can be rewritten as

$$
\left(e^{2 t}-1\right) \sum_{i=1}^{n}\left(\partial_{i} e^{-t \Delta} f\right)^{2} \leqslant \int_{0}^{t} \frac{\mathrm{d}}{\mathrm{d} s} e^{-s \Delta}\left(e^{-(t-s) \Delta} f\right)^{2} \mathrm{~d} s=e^{-t \Delta} f^{2}-\left(e^{-t \Delta} f\right)^{2} .
$$

Therefore,

$$
\sqrt{e^{2 t}-1}\left\|\nabla e^{-t \Delta} f\right\|_{L_{\infty}\left(\{-1,1\}^{n} ; \mathbb{C}\right)} \leqslant\left\|e^{-t \Delta} f^{2}\right\|_{L_{\infty}\left(\{-1,1\}^{n} ; \mathbb{C}\right)}^{1 / 2} \leqslant\|f\|_{L_{\infty}\left(\{-1,1\}^{n} ; \mathbb{C}\right)},
$$

which completes the proof of the lemma. 
Remark 48. In fact, it follows from (227) that for every scalar valued function $f:\{-1,1\}^{n} \rightarrow \mathbb{C}$ and $p \in[2, \infty]$, we have the inequality

$$
\forall t>0, \quad\left\|\nabla e^{-t \Delta} f\right\|_{L_{p}\left(\{-1,1\}^{n} ; \mathbb{C}\right)} \leqslant \frac{1}{\sqrt{e^{2 t}-1}}\|f\|_{L_{p}\left(\{-1,1\}^{n} ; \mathbb{C}\right)} .
$$

Combined with Lemma 44, these estimates provide the sharp dimension free decay of the operator norm of $\nabla e^{-t \Delta}$ from $L_{p}\left(\{-1,1\}^{n} ; \mathbb{C}\right)$ to itself for every $p \in(1, \infty]$.

Proof of Proposition 46. Fix $n \in \mathbb{N}, d \in\{1, \ldots, n\}$ and let $f:\{-1,1\}^{n} \rightarrow \mathbb{C}$ be a function of degree at most $d$. Then, by Lemma 47, Theorem 1 and the estimate $T_{d}\left(e^{t}\right) \leqslant e^{t d^{2}}$, we have

$$
\|\nabla f\|_{L_{\infty}\left(\{-1,1\}^{n} ; \mathbb{C}\right)} \leqslant \frac{1}{\sqrt{e^{2 t}-1}}\left\|e^{t \Delta} f\right\|_{L_{\infty}\left(\{-1,1\}^{n} ; \mathbb{C}\right)} \leqslant \frac{e^{t d^{2}}}{\sqrt{e^{2 t}-1}}\|f\|_{L_{\infty}\left(\{-1,1\}^{n} ; \mathbb{C}\right)} .
$$

Plugging $t=\frac{1}{d^{2}}$ and using that $\sqrt{e^{2 t}-1} \geqslant \sqrt{2 t}$, we thus get

$$
\|\nabla f\|_{L_{\infty}\left(\{-1,1\}^{n} ; \mathbb{C}\right)} \leqslant \frac{e d}{\sqrt{2}}\|f\|_{L_{\infty}\left(\{-1,1\}^{n} ; \mathbb{C}\right)}<2 d\|f\|_{L_{\infty}\left(\{-1,1\}^{n} ; \mathbb{C}\right)} .
$$

To prove that a $O(d)$ factor is necessary, consider the function $f:\{-1,1\}^{n} \rightarrow \mathbb{C}$ given by

$$
\forall \varepsilon=\left(\varepsilon_{1}, \ldots, \varepsilon_{n}\right) \in\{-1,1\}^{n}, \quad f(\varepsilon) \stackrel{\text { def }}{=} T_{d}\left(\frac{\varepsilon_{1}+\cdots+\varepsilon_{n}}{n}\right),
$$

where $T_{d}(x)$ is the $d$-th Chebyshev polynomial of the first kind. Clearly $f$ has degree at most $d$ and furthermore

$$
\|f\|_{L_{\infty}\left(\{-1,1\}^{n} ; \mathbb{C}\right)}=\max _{\varepsilon \in\{-1,1\}^{n}}\left|T_{d}\left(\frac{\varepsilon_{1}+\cdots+\varepsilon_{n}}{n}\right)\right|=T_{d}(1)=1
$$

On the other hand,

$$
\|\nabla f\|_{L_{\infty}\left(\{-1,1\}^{n} ; \mathbb{C}\right)} \geqslant\left(\sum_{i=1}^{n}\left(\partial_{i} f(1, \ldots, 1)\right)^{2}\right)^{1 / 2}
$$

and for every $i \in\{1, \ldots, n\}$,

$$
\partial_{i} f(1, \ldots, 1)=\frac{1}{2}\left(1-T_{d}\left(1-\frac{2}{n}\right)\right)
$$

Therefore,

$$
\|\nabla f\|_{L_{\infty}\left(\{-1,1\}^{n} ; \mathbb{C}\right)} \geqslant \frac{\sqrt{n}}{2}\left(1-T_{d}\left(1-\frac{2}{n}\right)\right)
$$

which for $n=d^{2}$ becomes

$$
\|\nabla f\|_{L_{\infty}\left(\{-1,1\}^{n} ; \mathbb{C}\right)} \geqslant \frac{d}{2}\left(1-T_{d}\left(1-\frac{2}{d^{2}}\right)\right) .
$$

Finally notice that

$$
1-T_{d}\left(1-\frac{2}{d^{2}}\right)=1-\cos \left(d \arccos \left(1-\frac{2}{d^{2}}\right)\right) \geqslant 1
$$

for large enough values of $d$, since

$$
\lim _{d \rightarrow \infty} 1-\cos \left(d \arccos \left(1-\frac{2}{d^{2}}\right)\right)=1-\cos (2) .
$$

The asymptotic optimality of (219) follows from (237) and (238). 
Remark 49. Using Theorem 13, one can also derive an endpoint Bernstein-Markov inequality for $p=1$. Indeed, using Jensen's inequality and orthogonality, for every $n, d \in \mathbb{N}$ with $d \in\{1, \ldots, n\}$, every function $f:\{-1,1\}^{n} \rightarrow \mathbb{C}$ satisfies

$$
\|\nabla f\|_{L_{1}\left(\{-1,1\}^{n} ; \mathbb{C}\right)} \leqslant\|\nabla f\|_{L_{2}\left(\{-1,1\}^{n} ; \mathbb{C}\right)} \leqslant \sqrt{d}\|f\|_{L_{2}\left(\{-1,1\}^{n} ; \mathbb{C}\right)}
$$

and invoking Theorem 13 we conclude that

$$
\|\nabla f\|_{L_{1}\left(\{-1,1\}^{n} ; \mathbb{C}\right)} \leqslant(2.69076)^{d} \sqrt{d}\|f\|_{L_{1}\left(\{-1,1\}^{n} ; \mathbb{C}\right)} .
$$

It would be interesting if one could obtain a polynomial bound in this inequality.

Remark 50. In our recent paper [EI18], we investigated Bernstein-Markov inequalities in the spirit of Theorem 15 for polynomials on $\mathbb{R}^{n}$ equipped with the Gaussian measure. The main tools we used were Epperson's Gaussian complex hypercontractivity [Epp89] (see also [Jan97]) and Meyer's Gaussian Riesz transform inequalities [Mey84]. Even though in [EI18] we did not consider vector valued inequalities, we note that all the results of Section 3 also hold true in the Gaussian setting by the holomorphicity of the Ornstein-Uhlenbeck semigroup $e^{-t L}$ on $K$-convex targets which follows from Pisier's $K$-convexity theorem [Pis82]. Moreover, it has been shown by Pisier [Pis88] that for every UMD Banach space $\left(X,\|\cdot\|_{X}\right)$ and $p \in(1, \infty)$ there exist $c(p, X), C(p, X) \in(0, \infty)$ such that for every $n \in \mathbb{N}$ and every function $f: \mathbb{R}^{n} \rightarrow X$, we have

$$
\begin{aligned}
& c(p, X)\left\|L^{1 / 2} f\right\|_{L_{p}\left(\left(\mathbb{R}^{n}, \gamma_{n}\right) ; X\right)} \leqslant\left(\int_{\mathbb{R}^{n}}\left\|\sum_{i=1}^{n} y_{i} \partial_{i} f\right\| L_{p}\left(\left(\mathbb{R}^{n}, \gamma_{n}\right) ; X\right)\right. \\
&\left.\mathrm{d} \gamma_{n}(y)\right)^{1 / p} \\
& \leqslant C(p, X)\left\|L^{1 / 2} f\right\|_{L_{p}\left(\left(\mathbb{R}^{n}, \gamma_{n}\right) ; X\right)} .
\end{aligned}
$$

Therefore, since UMD spaces have non-trivial type (e.g. by [Pis73]), we then conclude that for every $p \in(1, \infty)$ there exist $\beta=\beta(p, X) \in[0,1)$ and $K=K(p, X) \in(0, \infty)$ such that if $P$ is a polynomial on $\mathbb{R}^{n}$ of degree at most $d$, then

$$
\|\nabla P\|_{L_{p}\left(\left(\mathbb{R}^{n}, \gamma_{n}\right) ; X\right)} \stackrel{\text { def }}{=}\left(\int_{\mathbb{R}^{n}}\left\|\sum_{i=1}^{n} y_{i} \partial_{i} f\right\|_{L_{p}\left(\left(\mathbb{R}^{n}, \gamma_{n}\right) ; X\right)} \mathrm{d} \gamma_{n}(y)\right)^{1 / p} \leqslant K d^{\beta}\|P\|_{L_{p}\left(\left(\mathbb{R}^{n}, \gamma_{n}\right) ; X\right)} .
$$

Such a result for UMD-valued functions on the Hamming cube is unknown. Inequality (243) is a high dimensional vector valued analogue of Freud's inequality [Fre71], who showed (243) for $X=\mathbb{C}$ and $n=1$ with the optimal exponent $\beta=\frac{1}{2}$ for every $p \in[1, \infty)$. To the best of our knowledge no vector valued versions of Freud's inequality (even for $n=1$ ) were available in the literature.

We will now prove Corollary 16, the improved estimate on the influences of bounded functions. We will need the following lemma, whose proof relies on an inequality of Sarantopoulos [Sar91].

Lemma 51. Let $n \in \mathbb{N}, d \in\{1, \ldots, n\}$ and $p \in[1, \infty)$. Then, for every function $f:\{-1,1\}^{n} \rightarrow \mathbb{C}$ of degree at most $d$, we have

$$
\operatorname{Inf}^{(p)} f \leqslant \inf _{t>0}\left(\sup _{\operatorname{deg}(g) \leqslant d} \frac{\|g\|_{L_{p}\left(\{-1,1\}^{n} ; \mathbb{C}\right)}^{p}}{\left\|e^{-t \Delta} g\right\|_{L_{p}\left(\{-1,1\}^{n} ; \mathbb{C}\right)}^{p}}\right) \frac{d}{\sqrt{e^{2 t}-1}}\|f\|_{L_{\infty}\left(\{-1,1\}^{n} ; \mathbb{C}\right)^{\prime}}^{p} .
$$

Proof. For $t>0$, denote by

$$
C_{d}(t) \stackrel{\text { def }}{=} \sup _{\operatorname{deg}(g) \leqslant d} \frac{\|g\|_{L_{p}\left(\{-1,1\}^{n} ; \mathbb{C}\right)}^{p}}{\left\|e^{-t \Delta} g\right\|_{L_{p}\left(\{-1,1\}^{n} ; \mathbb{C}\right)}^{p}}
$$

and notice that

$$
\operatorname{Inf}^{(p)} f=\sum_{i=1}^{n}\left\|\partial_{i} f\right\|_{L_{p}\left(\{-1,1\}^{n} ; \mathbb{C}\right)}^{p} \leqslant C_{d}(t) \sum_{i=1}^{n}\left\|\partial_{i} e^{-t \Delta} f\right\|_{L_{p}\left(\{-1,1\}^{n} ; \mathbb{C}\right)}^{p}
$$


Moreover, we have

$$
\begin{aligned}
\sum_{i=1}^{n}\left\|\partial_{i} e^{-t \Delta} f\right\|_{L_{p}\left(\{-1,1\}^{n} ; \mathbb{C}\right)}^{p} \leqslant\left(\sum_{i=1}^{n}\left\|\partial_{i} e^{-t \Delta} f\right\|_{L_{1}\left(\{-1,1\}^{n} ; \mathbb{C}\right)}\right)\left\|e^{-t \Delta} f\right\|_{L_{\infty}\left(\{-1,1\}^{n} ; \mathbb{C}\right)}^{p-1} & \leqslant\|f\|_{L_{\infty}\left(\{-1,1\}^{n} ; \mathbb{C}\right)}^{p-1} \operatorname{Inf}^{(1)}\left(e^{-t \Delta} f\right)
\end{aligned}
$$

where the last inequality follows from the contractivity of the heat semigroup. Therefore, it suffices to show that

$$
\operatorname{Inf}^{(1)}\left(e^{-t \Delta} f\right) \leqslant \frac{d}{\sqrt{e^{2 t}-1}}\|f\|_{L_{\infty}\left(\{-1,1\}^{n} ; \mathbb{C}\right)} .
$$

Let $F\left(x_{1}, \ldots, x_{n}\right)$ be the multilinear polynomial on $\mathbb{R}^{n}$ given by

$$
\forall\left(x_{1}, \ldots, x_{n}\right) \in \mathbb{R}^{n}, \quad F\left(x_{1}, \ldots, x_{n}\right) \stackrel{\text { def }}{=} \sum_{A \subseteq\{1, \ldots, n\}} \widehat{f}(A) \prod_{i \in A} x_{i} .
$$

Notice that for $\varepsilon \in\{-1,1\}^{n}$, we have $e^{-t \Delta} f(\varepsilon)=F\left(e^{-t} \varepsilon\right)$ and for $i \in\{1, \ldots, n\},\left|\partial_{i} e^{-t \Delta} f(\varepsilon)\right|=$ $e^{-t}\left|\partial_{i} F(\varepsilon)\right|$, where $\partial_{i} F$ is the usual partial derivative of $F$. Consequently,

$$
\begin{aligned}
\operatorname{Inf}^{(1)}\left(e^{-t \Delta} f\right)=\frac{1}{2^{n}} & \sum_{\varepsilon \in\{-1,1\}^{n}} \sum_{i=1}^{n}\left|\partial_{i} e^{-t \Delta} f(\varepsilon)\right| \leqslant e^{-t} \max _{\varepsilon \in\{-1,1\}^{n}} \sum_{i=1}^{n}\left|\partial_{i} F\left(e^{-t} \varepsilon\right)\right| \\
& =e^{-t} \max _{\varepsilon, \delta \in\{-1,1\}^{n}} \sum_{i=1}^{n} \delta_{i} \partial_{i} F\left(e^{-t} \varepsilon\right)=e^{-t} \max _{\|x\|_{\infty} \leqslant e^{-t},\|y\|_{\infty} \leqslant 1} \sum_{i=1}^{n} y_{i} \partial_{i} F(x),
\end{aligned}
$$

where in the last equality we used the multilinearity of the polynomial $\sum_{i=1}^{n} y_{i} \partial_{i} F(x)$ in the variables $(x, y) \in \mathbb{R}^{2 n}$. By Sarantopoulos' vector valued Bernstein inequality from [Sar91], we deduce

$$
\max _{\|x\|_{\infty} \leqslant e^{-t},\|y\|_{\infty} \leqslant 1} \sum_{i=1}^{n} y_{i} \partial_{i} F(x) \leqslant \frac{d}{\sqrt{1-e^{-2 t}}} \max _{\|x\|_{\infty} \leqslant 1}|F(x)| \leqslant \frac{d}{\sqrt{1-e^{-2 t}}}\|f\|_{L_{\infty}\left(\{-1,1\}^{n} ; \mathbb{C}\right)},
$$

again by the multilinearity of $F$. Therefore, (250) and (251) imply (248), which combined with (246) and (247) completes the proof of the lemma.

Equipped with Lemma 51, we can prove Corollary 16.

Proof of Corollary 16. Fix $n \in \mathbb{N}, d \in\{1, \ldots, n\}, p \in\left(1, \frac{4}{3}\right)$ and let $f:\{-1,1\}^{n} \rightarrow \mathbb{C}$ be a function of degree at most $d$. Denote by

$$
\eta_{p} \stackrel{\text { def }}{=} \frac{\pi}{2 \pi-2 \arcsin \left(\frac{2 \sqrt{p-1}}{p}\right)} .
$$

It follows from (23) and (138) that there exists a constant $C_{p} \in(0, \infty)$ such that for every function $g:\{-1,1\}^{n} \rightarrow \mathbb{C}$ be a function of degree at most $d$,

$$
\forall 0 \leqslant t \leqslant 1, \quad\left\|e^{-t \Delta} g\right\|_{L_{p}\left(\{-1,1\}^{n} ; \mathbb{C}\right)} \geqslant e^{-C_{p} t^{\eta_{p}}} d\|g\|_{L_{p}\left(\{-1,1\}^{n} ; \mathbb{C}\right)} .
$$

Therefore, by Lemma 51, we conclude that

$$
\operatorname{Inf}^{(p)} f \leqslant \inf _{t>0} \frac{e^{p C_{p} t^{\eta p} d} d}{\sqrt{e^{2 t}-1}} \cdot\|f\|_{L_{\infty}\left(\{-1,1\}^{n} ; \mathbb{C}\right)}^{p} .
$$

Choosing $t=d^{-1 / \eta_{p}}$ and using that $\sqrt{e^{2 t}-1} \geqslant \sqrt{2 t}$, we deduce that there exists a constant $K_{p} \in(0, \infty)$ such that

$$
\operatorname{Inf}^{(p)} f \leqslant K_{p} d^{1+\frac{1}{2 \eta_{p}}}\|f\|_{L_{\infty}\left(\{-1,1\}^{n} ; \mathbb{C}\right)}^{p}=K_{p} d^{2-\frac{1}{\pi} \arcsin \left(\frac{2 \sqrt{p-1}}{p}\right)}\|f\|_{L_{\infty}\left(\{-1,1\}^{n} ; \mathbb{C}\right)}^{p},
$$

which concludes the proof. 
Finally, we present the proof of Theorem 17.

Proof of Theorem 17. Fix $n, d, m \in \mathbb{N}$ with $d+m \leqslant n$ and let $f:\{-1,1\}^{n} \rightarrow \mathbb{C}$ be a function of degree at most $d+m$ which is also in the $d$-th tail space. Then, by Lust-Piquard's lower Riesz transform inequalities [LP98], for every $p \in(1, \infty)$ there exists $c_{p} \in(0, \infty)$ such that

$$
\|\nabla f\|_{L_{p}\left(\{-1,1\}^{n} ; \mathbb{C}\right)} \stackrel{(200)}{\geqslant} c_{p}\left\|\Delta^{1 / 2} f\right\|_{L_{p}\left(\{-1,1\}^{n} ; \mathbb{C}\right)} .
$$

The conclusion of the theorem now follows from (159).

\section{REFERENCES}

[AA14] S. Aaronson and A. Ambainis. The need for structure in quantum speedups. Theory Comput., 10:133-166, 2014.

[BB14] A. Bačkurs and M. Bavarian. On the sum of $L_{1}$ influences. In IEEE 29th Conference on Computational Complexity - CCC 2014, pages 132-143. IEEE Computer Soc., Los Alamitos, CA, 2014.

[BE95] P. Borwein and T. Erdélyi. Polynomials and polynomial inequalities, volume 161 of Graduate Texts in Mathematics. Springer-Verlag, New York, 1995.

[Bec75] W. Beckner. Inequalities in Fourier analysis. Ann. of Math. (2), 102(1):159-182, 1975.

[BELP08] L. Ben Efraim and F. Lust-Piquard. Poincaré type inequalities on the discrete cube and in the CAR algebra. Probab. Theory Related Fields, 141(3-4):569-602, 2008.

[BGL14] D. Bakry, I. Gentil, and M. Ledoux. Analysis and geometry of Markov diffusion operators, volume 348 of Grundlehren der Mathematischen Wissenschaften. Springer, Cham, 2014.

[Bon70] A. Bonami. Étude des coefficients de Fourier des fonctions de $L^{p}(G)$. Ann. Inst. Fourier (Grenoble), 20(fasc. 2):335-402 (1971), 1970.

[Bor79] C. Borell. On the integrability of Banach space valued Walsh polynomials. In Séminaire de Probabilités, XIII, volume 721 of Lecture Notes in Math., pages 1-3. Springer, Berlin, 1979.

[Bor84] C. Borell. On polynomial chaos and integrability. Probab. Math. Statist., 3(2):191-203, 1984.

[Bou80] J. Bourgain. Walsh subspaces of $L^{p}$-product spaces. In Seminar on Functional Analysis, 1979-1980 (French), pages Exp. No. 4A, 9. École Polytech., Palaiseau, 1980.

[EI18] A. Eskenazis and P. Ivanisvili. Dimension independent Bernstein-Markov inequalities in Gauss space. To appear in J. Approx. Theory. Preprint available at https://arxiv.org/abs/1808.01273, 2018.

[EN20] A. Eskenazis and A. Naor. Discrete Littlewood-Paley-Stein theory and Pisier's inequality for superreflexive targets. Preprint, 2020.

[Epp89] J. B. Epperson. The hypercontractive approach to exactly bounding an operator with complex Gaussian kernel. J. Funct. Anal., 87(1):1-30, 1989.

[Erd20] T. Erdélyi. Reverse Markov- and Bernstein-type inequalities for incomplete polynomials. J. Approx. Theory, 251:105341, 2020.

$\left[\mathrm{FHH}^{+}\right.$14] Y. Filmus, H. Hatami, S. Heilman, E. Mossel, R. O’Donnell, S. Sachdeva, A. Wan, and K. Wimmer. Real Analysis in Computer Science: a collection of open problems. Preprint available at https://simons . berkeley.edu/sites/default/files/openprobsmerged.pdf, 2014.

[FHKL16] Y. Filmus, H. Hatami, N. Keller, and N. Lifshitz. On the sum of the $L_{1}$ influences of bounded functions. Israel J. Math., 214(1):167-192, 2016.

[Fre71] G. Freud. A certain inequality of Markov type. Dokl. Akad. Nauk SSSR, 197:790-793, 1971.

[HMO17] S. Heilman, E. Mossel, and K. Oleszkiewicz. Strong contraction and influences in tail spaces. Trans. Amer. Math. Soc., 369(7):4843-4863, 2017.

[HN13] T. Hytönen and A. Naor. Pisier's inequality revisited. Studia Math., 215(3):221-235, 2013.

[IN19] P. Ivanisvili and F. Nazarov. On Weissler's conjecture on the Hamming cube I. Preprint available at https://arxiv.org/abs/1907.11359, 2019.

[IT19] P. Ivanisvili and T. Tkocz. Comparison of moments of Rademacher chaoses. Ark. Mat., 57(1):121-128, 2019 .

[Jan97] S. Janson. On complex hypercontractivity. J. Funct. Anal., 151(1):270-280, 1997.

[Kah64] J.-P. Kahane. Sur les sommes vectorielles $\sum \pm u_{n}$. C. R. Acad. Sci. Paris, 259:2577-2580, 1964.

[Khi23] A. Khintchine. Über dyadische Brüche. Math. Z., 18(1):109-116, 1923.

[Kra06] S. G. Krantz. Geometric function theory. Cornerstones. Birkhäuser Boston, Inc., Boston, MA, 2006. Explorations in complex analysis.

[KW92] S. Kwapień and W. A. Woyczyński. Random series and stochastic integrals: single and multiple. Probability and its Applications. Birkhäuser Boston, Inc., Boston, MA, 1992. 
[Kwa76] S. Kwapień. A theorem on the Rademacher series with vector valued coefficients. In Probability in Banach spaces, pages 157-158. Lecture Notes in Math., Vol. 526. Springer, Berlin, 1976.

[LP98] F. Lust-Piquard. Riesz transforms associated with the number operator on the Walsh system and the fermions. J. Funct. Anal., 155(1):263-285, 1998.

[Mau03] B. Maurey. Type, cotype and K-convexity. In Handbook of the geometry of Banach spaces, Vol. 2, pages 1299-1332. North-Holland, Amsterdam, 2003.

[Mey84] P.-A. Meyer. Transformations de Riesz pour les lois gaussiennes. In Seminar on probability, XVIII, volume 1059 of Lecture Notes in Math., pages 179-193. Springer, Berlin, 1984.

[MN14] M. Mendel and A. Naor. Nonlinear spectral calculus and super-expanders. Publ. Math. Inst. Hautes Études Sci., 119:1-95, 2014.

[MP76] B. Maurey and G. Pisier. Séries de variables aléatoires vectorielles indépendantes et propriétés géométriques des espaces de Banach. Studia Math., 58(1):45-90, 1976.

[MS86] V. D. Milman and G. Schechtman. Asymptotic theory of finite-dimensional normed spaces, volume 1200 of Lecture Notes in Mathematics. Springer-Verlag, Berlin, 1986. With an appendix by M. Gromov.

[Nao12] A. Naor. An introduction to the Ribe program. Jpn. J. Math., 7(2):167-233, 2012.

[NS02] A. Naor and G. Schechtman. Remarks on non linear type and Pisier's inequality. J. Reine Angew. Math., 552:213-236, 2002.

[O'D14] R. O'Donnell. Analysis of Boolean functions. Cambridge University Press, New York, 2014.

[Ole03] K. Oleszkiewicz. On a nonsymmetric version of the Khinchine-Kahane inequality. In Stochastic inequalities and applications, volume 56 of Progr. Probab., pages 157-168. Birkhäuser, Basel, 2003.

[Pis73] G. Pisier. Sur les espaces de Banach qui ne contiennent pas uniformément de $l_{n}^{1}$. C. R. Acad. Sci. Paris Sér. A-B, 277:A991-A994, 1973.

[Pis78] G. Pisier. Les inégalités de Khintchine-Kahane, d'après C. Borell. In Séminaire sur la Géométrie des Espaces de Banach (1977-1978), pages Exp. No. 7, 14. École Polytech., Palaiseau, 1978.

[Pis82] G. Pisier. Holomorphic semigroups and the geometry of Banach spaces. Ann. of Math. (2), 115(2):375-392, 1982.

[Pis86] G. Pisier. Probabilistic methods in the geometry of Banach spaces. In Probability and analysis (Varenna, 1985), volume 1206 of Lecture Notes in Math., pages 167-241. Springer, Berlin, 1986.

[Pis88] G. Pisier. Riesz transforms: a simpler analytic proof of P.-A. Meyer's inequality. In Séminaire de Probabilités, XXII, volume 1321 of Lecture Notes in Math., pages 485-501. Springer, Berlin, 1988.

[Pis07] G. Pisier. A remark on hypercontractive semigroups and operator ideals. Preprint available at https: //arxiv.org/abs/0708.3423, 2007.

[Sar91] Y. Sarantopoulos. Bounds on the derivatives of polynomials on Banach spaces. Math. Proc. Cambridge Philos. Soc., 110(2):307-312, 1991.

[Ste70] E. M. Stein. Topics in harmonic analysis related to the Littlewood-Paley theory. Annals of Mathematics Studies, No. 63. Princeton University Press, Princeton, N.J.; University of Tokyo Press, Tokyo, 1970.

[Sze25] G. Szegö. Über einen Satz von A. Markoff. Math. Z., 23(1):45-61, 1925.

[Tal93] M. Talagrand. Isoperimetry, logarithmic Sobolev inequalities on the discrete cube, and Margulis' graph connectivity theorem. Geom. Funct. Anal., 3(3):295-314, 1993.

[Wag00] R. Wagner. Notes on an inequality by Pisier for functions on the discrete cube. In Geometric aspects of functional analysis, volume 1745 of Lecture Notes in Math., pages 263-268. Springer, Berlin, 2000.

[Wei79] F. B. Weissler. Two-point inequalities, the Hermite semigroup, and the Gauss-Weierstrass semigroup. J. Funct. Anal., 32(1):102-121, 1979.

[Wol07] P. Wolff. Hypercontractivity of simple random variables. Studia Math., 180(3):219-236, 2007.

(A. E.) Department of Mathematics, Princeton University, Princeton, NJ 08544-1000, USA

E-mail address: ae3@math.princeton.edu

(P. I.) Department of Mathematics, University of California, Irvine, CA 92617, USA

E-mail address: pivanisv@uci.edu 